Livraisons

d'Histoire

de l'Architecture

\section{Livraisons de l'histoire de l'architecture}

27 | 2014

Varia II

\title{
Paul Tournon (1881 - 1964), un architecte catholique
}

Paul Tournon (1881-1964), a catholic architect

Paul Tournon (1881-1964), ein katholischer Architekt

\section{Miriam Simon}

\section{OpenEdition}

Journals

Édition électronique

URL : http://journals.openedition.org/lha/366

DOI : 10.4000/lha.366

ISSN : 1960-5994

Éditeur

Association Livraisons d'histoire de l'architecture - LHA

Édition imprimée

Date de publication : 10 juin 2014

Pagination : $75-116$

ISSN : 1627-4970

Référence électronique

Miriam Simon, « Paul Tournon (1881 - 1964), un architecte catholique », Livraisons de l'histoire de l'architecture [En ligne], 27 | 2014, mis en ligne le 10 juin 2016, consulté le 30 avril 2019. URL : http:// journals.openedition.org/lha/366 ; DOI : 10.4000/lha.366 
Par Miriam SIMON

\section{PAUL TOURNON (1881-1964), UN ARCHITECTE CATHOLIQUE ${ }^{1}$}

Le chantier de l'église du Saint-Esprit (ill. 1), la « reine des Églises du cardinal ${ }^{2}$, apporte un éclairage riche sur les conceptions de Paul Tournon dans ses choix stylistiques, constructifs et spirituels, tant la construction d'églises constitue une part centrale du corpus d'un architecte qui déclare avoir toujours été attiré par l'architecture sacrée. L'inventaire du décor, effectué en $2000^{3}$, s'appuie sur un dépouillement partiel des archives professionnelles de Paul Tournon, données aux Archives nationales par ses filles en $1977^{4}$. La consultation du fonds remis à l'Académie d'architecture ${ }^{5}$, des archives de la Conservation des œuvres d'art religieuses et civiles, de celles de l'Archevêché de Paris et des Archives de Paris ainsi que de revues contemporaines, vient compléter ces deux sources principales.

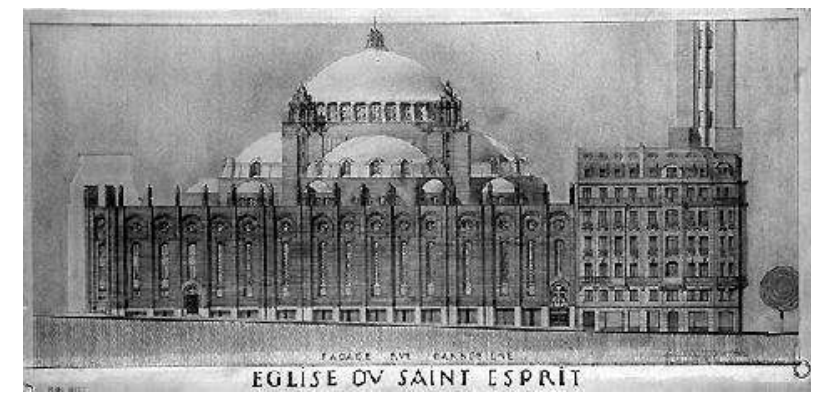

Ill. 1 : Paul Tournon, Église du Saint-Esprit, élévation de la façade rue Cannebière, 1928, mine de plomb sur carton, IFA, TOURN/D/28/1 351 AA 25 (photographie: Fouin Christophe () Ville de Paris, COARC, 2002. Base mémoire : DSC_0517.JPG).

1. Je remercie le P. Jacques Hadengue, Marion Tournon-Branly, Christophe Fouin, Jean-Charles Cappronnier, Cécile Souchon, Véronique David, Nadine Gastaldi, Isabelle Duhau, Marie-Laure Crosnier-Leconte, Micheline Silvestre, Geneviève Thory et Micheline Tissot.

2. R. L., "Première fête de la Pentecôte en la chapelle du Saint-Esprit ", La Semaine religieuse de Paris, 15 juin 1935, p. 886.

3. Inventaire effectué au sein de la conservation des œuvres d'art religieuses et civiles, direction des affaires culturelles de la Ville de Paris.

4. Marion et Florence Tournon-Branly. Ce fonds, rassemblé sous la cote Arch. nat. 377 AP 1-294, couvre une période qui s'étend de 1905 à 1964. Il se compose matériellement de 190 cartons, 75 rouleaux, 45 portefeuilles de plans, 4 cartons de catalogues, de documentation imprimée et photographique, et de la correspondance de l'agence.

Sauf mention contraire, les cotes indiquées en note concernent ce fonds.

5. Désormais déposé à l'Institut français d'architecture. 
Après l'analyse d'une biographie qui révèle un homme influent et actif dans de nombreuses instances, l'étude de la construction de l'église du Saint-Esprit s'attache à décrire une conception totalisante du décor de l'édifice, corollaire d'une pratique traditionnelle de la maîtrise d'œuvre. La place respective de la tradition et de la modernité dans l'architecture religieuse de Paul Tournon, est ensuite étudiée et mise en regard avec l'œuvre de Perret.

\section{Un homme influent}

Ce natif de Marseille s'est formé auprès de Gausendi Allar, ancien chef d'agence de l'architecte Espérandieu ${ }^{6}$, chez qui il avait suivi notamment la construction des coupoles de la cathédrale et de la basilique Notre-Dame de la Garde édifiée selon une inspiration néo-byzantine. Après trois ans d'études à l'École des Beaux-Arts de la capitale phocéenne, le jeune homme obtient une bourse d'étude lui permettant d'être admis en octobre 1902 à celle de Paris. Sa formation est couronnée par l'obtention du deuxième grand prix de Rome en 1911, ainsi que d'une bourse de voyage qui lui permet de visiter, en 1912, l'Allemagne, l'Italie, l'Espagne, l'Angleterre et la Hollande. Il ouvre une agence avec son confrère et compatriote Gaston Castel (1886-1971) mais sa carrière, interrompue par la guerre ${ }^{7}$, reprend après celle-ci avec la commande de nombreux monuments aux Morts, pour lesquels il s'adjoint le talent du sculpteur Antoine Sartorio (1885-1988). Issu d'une famille modeste, nourri par le sens de l'effort, Paul Tournon n'est pas insensible aux honneurs et recevra de nombreuses décorations françaises et étrangères. Son sens des relations humaines et sociales lui permet d'exercer de nombreuses responsabilités professionnelles. Reçu en 1913 au concours des Bâtiments civils et des Palais nationaux, membre temporaire du conseil général des bâtiments civils en 1930, Architecte en chef des bâtiments civils et des Palais nationaux depuis 1931, chargé de divers monuments historiques en 1932, des Archives nationales et de l'hôtel de Rohan en 1937, il devient architecte en chef de la Banque de France à partir de 1940, architecte-conseil de la Radiodiffusion nationale, et obtient la direction de la région d'Abbeville pour la seconde reconstruction. Parallèlement, se déroule une carrière académique exemplaire : il est élu membre de l'Institut en 1942, pour devenir président de l'Académie des Beaux-Arts en 1950.

Son activité d'enseignement commence en 1925, lorsqu'il est nommé chef d'un atelier d'architecture aux Beaux-Arts. Après l'armistice de 1940, Louis Hautecœur, secrétaire général aux Beaux-Arts lui confie la chaire de théorie de l'architecture à l'École des Beaux-Arts jusqu'en 1942, année au cours de laquelle il est nommé directeur (jusqu'en 1948) et directeur de l'École des arts décoratifs (jusqu'en 1944).

6. Henri-Jacques Espérandieu (1829-1874) termina les travaux de la cathédrale, la " Major ", commencée en 1852 par Léon Vaudoyer (1803-1872), et construisit le palais Longchamp. Son ouvre la plus populaire est la basilique Notre-Dame de la Garde, à laquelle il travailla de 1864 à sa mort. 7. Il servira sous les drapeaux et se verra décerner la croix de guerre en 1918. 
Tournon s'emploie alors à confier des travaux aux élèves pour leur épargner le service du travail obligatoire en Allemagne, trouvant des relevés à effectuer dans les villages de province ${ }^{8}$. Par-delà cette attitude paternelle, il défend ardemment l'École au travers de son principal fleuron, le concours de Rome, exaltant le caractère formateur de certaines contraintes techniques devenues incontournables au sortir de la seconde guerre mondiale et défendant les prérogatives de l'architecte face à l'ingénieur. En 1944, il s'oppose à la séparation de la section d'architecture du reste de l'enseignement de l'École des Beaux-Arts, ce qui «ne manquerait pas à bref délai de dessécher l'enseignement de cet art dont les attributions ne sauraient être réduites à l'art exclusif de bâtir "?

Tournon est un acteur important dans les instances officielles de la profession, qui se soucie de fédérer celle-ci. Membre de la société des architectes diplômés par le gouvernement et de la société des architectes modernes ${ }^{10}$, il a présidé la société centrale des architectes de 1945 à 1948. L'élimination immédiate du projet des frères Perret au concours lancé en 1926 pour la basilique Sainte-Jeanne d'Arc semble l'avoir affecté, de même que la reprise dans le programme imposé au second tour de sa propre idée de façade précédée d'une cour et d'un portique ${ }^{11}$ (ill. 2). Aussi se montre-t-il désireux, dès avant la seconde guerre mondiale, de voir créer un ordre des architectes. Sa réaction devant l'importance grandissante de Charles Venner, un ancien métreur dépourvu selon lui de compétences techniques mais qui se verra confier au sein des chantiers du cardinal la construction d'une vingtaine d'églises dans la banlieue parisienne, l'amène à écrire en 1941 à plusieurs membres de l'Institut pour demander la réactivation de la commission diocésaine d'architecture. Dans le prolongement d'un fonctionnement inauguré au XIX siècle, l'archevêché de Paris avait mis en place, après le vote de la loi de séparation des Églises et de l'État, cette commission dont l'activité devait encadrer sa politique de construction de nouveaux lieux de culte à la faveur de l'œuvre des chapelles de secours instituée en 1901. Progressivement, les tenants des Beaux-Arts y succédèrent aux rationalistes, à la faveur du remplacement de l'œuvre par les Chantiers du cardinal - ou Euvre des nouvelles paroisses de la région parisienne - au début de 1932.

8. Il suggère également de réaliser à l'Opéra des sculptures "en toc " en ouvrant un grand atelier à Versailles (Marion Tournon-Branly, entretien, 12 décembre 2003), propose un chantier-école dans la chapelle Saint-Symphorien à Saint-Germain-des-Prés (Arch. nat., 377 AP 189, 27 mai 1941).

9. Arch. nat., 377 AP 214, f 516, 16 octobre 1944, au directeur général des Beaux-Arts. C'est néanmoins le parti qui sera adopté à la fin des années 1960 .

10. Membre au moins depuis 1933 et trésorier de 1935 à 1946. Parmi les autres membres, citons Daniel Letrosne, Alfred-Donat Agache, Louis Sue, Albert Laprade, Éric Bagge, André Ventre, Louis Boileau, Joseph Marrast, Marcel Magne, Pierre-Anne Paquet, Hector Guimard, Henri Expert, Michel Roux-Spitz, Tony Garnier, Jacques Droz, Jean-Charles Moreux, Charles-Henri Besnard, Louis Debat-Ponsan, Georges Gaudibert, Georges Levard.

11. La raison officielle en étant que les Perret étaient aussi des entrepreneurs. Cf. Yvanhoé Rambosson : "Nécessité d'un "ordre des architectes", les suites des injustices du Concours Jeanne-d'Arc ", Comoedia, 15 novembre 1926 ; "Le concours pour la construction de l'église Sainte-Jeanne-d'Arc à Paris ", L'Architecture, vol. XL, nº 3. Marion Tournon-Branly, entretien, 12 décembre 2003. 


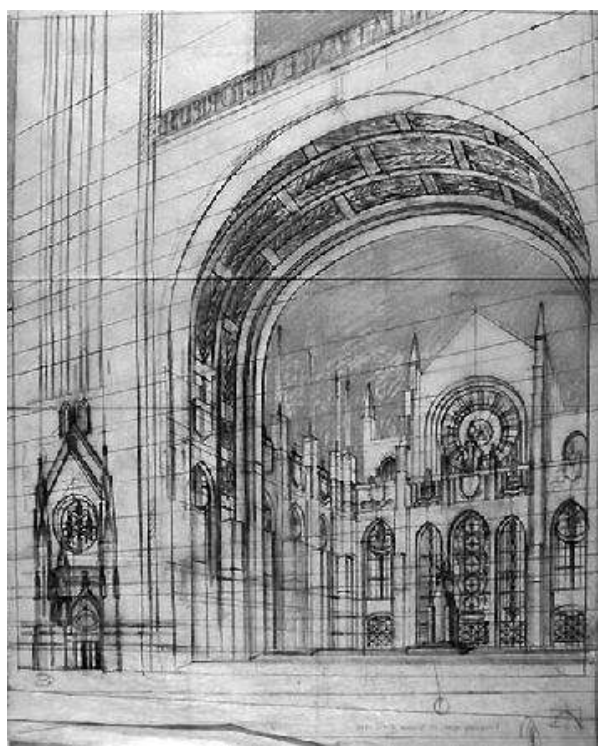

Ill. 2 : Paul Tournon, Projet pour la basilique Sainte-Jeanne-d'Arc, Vue en perspective du parvis, concours de 1926, fusain et crayon bleu sur papier calque, Arch. nat., 377 AP/240 i 2 10-102 (photographie : Fouin Christophe (C) Ville de Paris, COARC, 2002. Base mémoire : DSC_3369.JPG).

Tournon est également membre de plusieurs sociétés d'art et d'artistes : les Catholiques des Beaux-Arts, l'association Fra Angelico, la société des amis des cathédrales, la société de Saint-Jean, et s'implique énormément dans la vie des catholiques des Beaux-Arts - il y fait la connaissance de la fille du physicien Édouard Branly (1844-1940), Élisabeth (1889-1972), qu'il épouse en 1920 -, et y développe un esprit collectif jamais démenti. Il comprend rapidement l'importance de faire connaître son travail, par tous les moyens disponibles : organisation de visites de chantier, diffusion de photographies, participation à des expositions d'art sacré. L'exemple des visites du chantier de l'église du Saint-Esprit illustre le parti que sait en tirer l'architecte : Mgr Verdier s'y rend à plusieurs reprises en sa compagnie, notamment pour manifester l'intérêt porté par l'archevêché de Paris à cette construction. Il le fait visiter également aux Amis des cathédrales, ainsi qu’à certains confrères et ne répugne pas à y guider des journalistes, fournissant volontiers de la documentation à qui le lui demande, que ce soit pour illustrer la nouvelle revue Christ dans la banlieue, des revues d'art chrétien ou d'architecture. Cela vaudra à ce chantier une presse particulièrement abondante, dont les critiques modérés se montreront les plus élogieux (L'Architecture, La Construction Moderne, La Technique des Travaux).

Le corpus des commandes religieuses à Paris et en région parisienne est à la mesure de la forte implication de l'architecte dans le domaine religieux. Gros chantiers ou constructions économiques, Tournon intervient dans une large gamme de prix et de types d'églises - paroissiale, votive, officielle, pour le seul clocher ou 


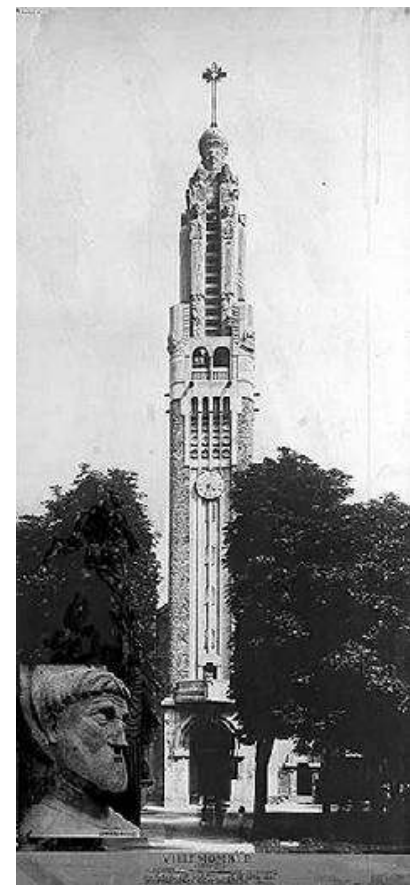

Ill. 3 : Vue latérale de l'église et du clocher Saint-Pierre de Villemomble, début du siècle, et 1926-1927, photographie après 1927. Cl. anonyme, IFA, TOURN/D/26/2 351 AA 20 (reproduction: Fouin Christophe (C) Ville de Paris, COARC, 2002. Base mémoire: DSC_0525.JPG).

simple chapelle. Après quelques commandes de moindre importance - l'autel de la chapelle Saint-François d'Assise dans la crypte du Sacré-Cœur de Montmartre avant-guerre, le maître-autel de l'église Saint-François-de-Sales de la rue Ampère (17 $7^{\mathrm{e}}$ arrondissement) au début des années Vingt -, sa rencontre avec le curé de Saint-Louis de Villemomble ouvre la période la plus innovante dans la carrière de Paul Tournon (ill. 3). Le projet pour le clocher, remis en août 1925, est réalisé en 1926 par le constructeur Fabre, en collaboration avec Carlo Sarrabezolles (18881971) pour le décor sculpté ${ }^{12}$. Un début de renommée lui vaut la commande en 1927 de la chapelle Sainte-Thérèse de l'Enfant-Jésus, à Aubergenville dans les Yvelines, pour la cité balnéaire d'Élisabethville ${ }^{13}$ (ill. 4). L'ossature en béton armé comprend une série de piliers extérieurs, entre lesquels sont tendus des entraits également reportés à l'extérieur de la voûte, à hauteur de clef. Les voûtes sont à

12. Édifiée au début du siècle, l'église Saint-Louis fut complétée en 1926-27 par la construction d'un clocher en ciment armé au milieu de la façade principale.

13. L’usage du béton armé est spécifié dans la commande. L'église d'Élisabethville, dotée d'un plan basilical à une nef, est fermée par d'importants vitraux de Marguerite Huré et bordée de petits bas-côtés. Cette "halle » est conçue comme un bâtiment-reliquaire, à l'instar de la Sainte-Chapelle de Paris. 


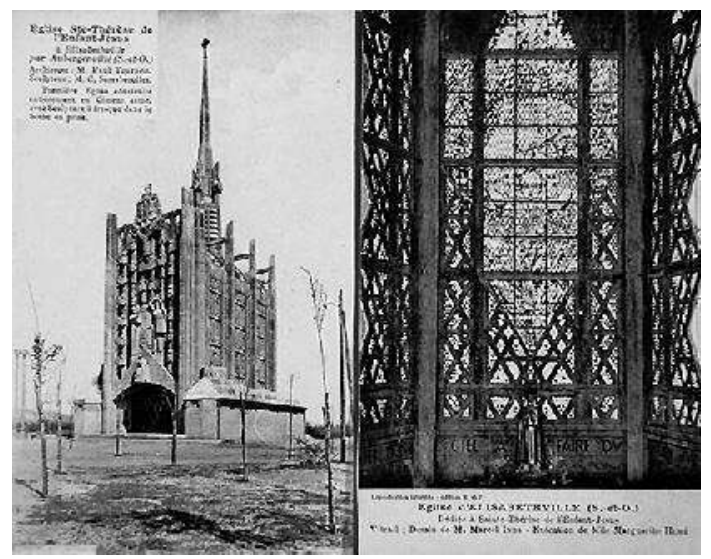

Ill. 4 : Église Sainte-Thérèse de l'Enfant-Jésus à Élisabethville, vues extérieure et intérieure, vers 1928. Photographe anonyme. Arch. nat., 377/AP-294 (photographie : Fouin Christophe (C) Ville de Paris, COARC, 2002. Base mémoire: DSC_8884.JPG).

doubles coques ventilées dans lesquelles sont incrustés des verres de couleur. Les sculptures du monument ont été conçues et exécutées par Sarrabezolles ${ }^{14}$.

L'église du Saint-Esprit lui avait été commandée dès $1926^{15}$. Sa construction s'échelonnera jusqu'en 1961. Les travaux de fondation coïncident en 1927 avec de premières études pour le Maroc et un projet pour Manizales, en Colombie. Suit la commande, pendant l'édification du gros œuvre, du Pavillon des missions pour l'Exposition coloniale de 1931. L'édifice principal de plan carré, couvert d'un toit en pagode évoquant l'Asie, est jouxté d'une construction basse et étroite précédée d'un avant-corps octogonal avec couverture de tuiles vertes, d'inspiration marocaine, et d'une tour revêtue d'un enduit à l'aspect de pisé rouge rappelant certaines constructions d'Afrique noire (ill. 5). Des études pour les Chantiers du Cardinal s'ensuivent en banlieue : Tournon travaille à des projets pour Orly (non réalisé), Châtillon-sous-Bagneux (non réalisé), La Garenne à Antony, Alfortville, La Briche à Épinay-sur-Seine (non réalisé) et Bobigny ${ }^{16}$. Dans tous les cas, l'architecte associe le béton pour l'ossature et le moellon ou la brique pour l'appareillage. NotreDame-des-Missions, qui dessert le Cygne-d'Enghien à Épinay-sur-Seine, est la reprise en dur en 1933 d'une partie du Pavillon des missions, avec tous les éléments du décor. Tournon est ultérieurement chargé de l'édification du Pavillon pontifical à l'Exposition internationale de 1937, sanctuaire à son tour reconstruit avec de légères variantes à Amiens, en 1957, sous le vocable de Saint-Honoré. Un projet pour un musée de la civilisation chrétienne enfin, établi en 1939-1940, ne verra jamais le jour.

14. Gwenaël Delhumeau, La Construction moderne, $\mathrm{n}^{\circ}$ 40, mars 1987.

15. L'Église du Saint-Esprit, Les Guides de l'Art sacré, s.d.

16. Cette dernière chapelle fut détruite par un incendie après 1979. Les Chantiers du cardinal, S.C.O.R.E. éd., s.d. [1979 ?], repr. p. 9; Jean-Pol Hindré, "Cinquante ans d'architecture religieuse ", Les Chantiers du cardinal, $\mathrm{n}^{\circ} 74$, juin 1981, p. 16-24, ill. p. 19. 


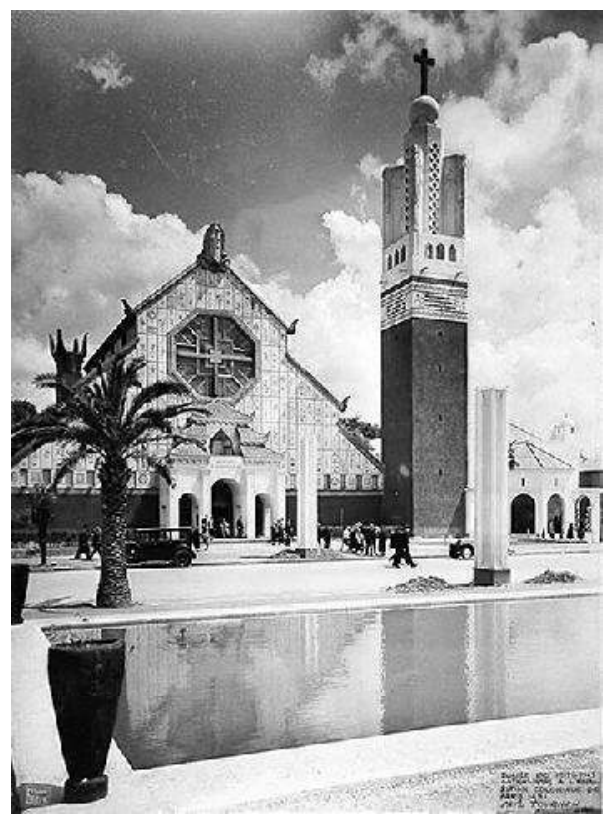

Ill. 5: Paul Tournon, Façades de l'église des missions catholiques à l'Exposition coloniale de 1931, positif sur verre. Arch. nat., $377 \mathrm{AP/}$ carton non coté (reproduction : Fouin Christophe (C) Ville de Paris, COARC, 2002. Base mémoire: DSC_8960.JPG).

\section{La reine des chantiers}

La commande de l'un des plus importants chantiers religieux parisiens de l'Entre-deux-guerres est à porter au crédit du cardinal Louis-Ernest Dubois (18561929), promoteur d'églises dont la gloire fut éclipsée par la forte médiatisation de l'œuvre des Chantiers du cardinal promue par son successeur, Mgr Jean Verdier (1864-1940 ${ }^{17}$. Le choix de la localisation relève d'une volonté de l'archevêché de procéder à un rééquilibrage du maillage paroissial en faveur de l'est parisien, dans un quartier qui connaît un fort accroissement de population après la première guerre mondiale. La paroisse est confiée aux Pères de la Miséricorde ${ }^{18}$, relayés par la congrégation des Eudistes fondée en 1643 par saint Jean-Eudes et qui s'orienta au XIX ${ }^{e}$ siècle vers l'enseignement secondaire, dans une perspective missionnaire expliquant l'attribution de cette paroisse, au vocable dédié au Saint-Esprit.

17. Conscient de l'immensité des besoins de son diocèse en matière d'équipement religieux, le cardinal Verdier lance en 1932 un emprunt destiné à financer un vaste programme de construction d'églises dans le diocèse de Paris. Il y intègre celle du Saint-Esprit, septième «Chantier du Cardinal ».

18. Présents lors de la cérémonie de pose de la première pierre : La Semaine religieuse de Paris, 16 juin 1928, rapporté dans L'Hirondelle de Saint-Camille, $\mathrm{n}^{\circ}$ 45, juillet 1928, p. 5. 
Les objectifs de l'archevêché - apostolat social, lutte contre le chômage dans un contexte de crise économique - se doublent dans le cas du Saint-Esprit d'un «apostolat artistique " ${ }^{19}$, les différents arts y trouvant un champ d'expression privilégié. Le programme prévoit une crypte, un presbytère, une église haute, des salles d'œuvres, un clocher habité, enfin des terrains de jeu et de détente en terrasse. La première pierre est posée le 2 juin 1928 et la crypte, inaugurée à la Pentecôte de l'année suivante. Les demi coupoles sont édifiées à l'automne 1931, et le gros œuvre achevé en 1933, hormis la façade de l'avenue Daumesnil. Le cardinal Verdier inaugure l'église haute le 23 décembre 1934. Quant au clocher et à la façade principale, leur construction sera reportée d'année en année, depuis 1933 jusqu'à la fin des années 1950, la porte principale n'étant commandée qu'en $1961^{20}$.

Les états de situation de 1932 donnent la mesure de l'ampleur du projet et renseignent sur la modestie de son coût au regard des surfaces prévues ${ }^{21}: 2450 \mathrm{~m}^{2}$ pour l'église, $850 \mathrm{~m}^{2}$ pour une crypte pouvant contenir 1500 fidèles. À cette date, le coût total devait s'élever, de manière prévisionnelle, à un montant de dix millions de francs, décor compris - mobilier fixe de la crypte, aménagement et décor de l'église haute ${ }^{22}$. L'échelonnement des travaux dans le temps rendit nécessaires plusieurs réévaluations.

L'importance des volumes conditionne le choix de la structure et des matériaux. L'ossature de l'édifice est en béton armé, revêtu de briques appareillées à l'extérieur, d'un remplissage en meulière pour les parties cachées, le béton étant laissé brut de décoffrage à l'intérieur - hormis pour la crypte et le narthex, enduits de ciment ${ }^{23}$. Le parti constructif est mis en évidence : la coupole centrale - culminant à une hauteur de 33 mètres, d'un diamètre de 22 mètres, armée de méridiens et de parallèles d'acier - forme un monolithe sans poussées reposant sur ses seuls points d'appui. Elle est néanmoins encadrée de quatre demi coupoles, elles-mêmes contrebutées chacune par trois coupoles plus petites. La partie centrale est séparée des bas-côtés par des arcades supportant une imposante galerie de passage aux allures d'aqueduc romain. Constituée de trois parties - une calotte armée portante, un revêtement de "corps creux Roger ", enfin une deuxième calotte armée de protection $^{24}$-, la coupole centrale est réalisée en vingt-sept jours, tour de force salué par

19. Charles Imbert, "L'Église du Saint-Esprit à Paris », La Technique des Travaux, nº 3, mars 1933, p. 132.

20. Archives de la Conservation des œuvres d'art religieuses et civiles : la cession de l'église à la Ville de Paris est signée le 27 décembre 1942, à charge pour celle-ci d'achever la construction, y compris celle du presbytère. Sur la construction du clocher, de 1957 à 1959, cf. archives COARC, SaintEsprit.

21. Arch. nat., 377 AP 85 , dossier T, s.d.

22. Arch. nat., 377 AP 275, f 70, 26 janvier 1932, à Mgr Crépin.

23. Le mobilier de la crypte, en partie fixe, est constitué d'un autel majeur en pierre, ciment et mosaïque, de sièges de célébrants et de stalles; les autels latéraux et les deux ambons sont ornés de sculptures de Sarrabezolles taillées à frais dans le béton. Les vitraux sont de Louzier, sur des cartons de Marcel Imbs.

24. Corps creux Roger : hourdis intermédiaire en terre cuite formant matelas d'air, destiné à atténuer les différences de température. Arch. nat., 377 AP 201, f 425, 17 juin 1935, Recommandation. 
la presse. Des contraintes économiques serrées interdiront à Tournon de la protéger au moyen d'une couverture de cuivre, et il aura recours à l'expédient, inefficace, d'un revêtement du dôme au moyen de trois couches de Stic B ${ }^{25}$. Le chœur et le ciborium, construits à l'automne 1934, possèdent également une ossature en béton armé. De part et d'autre du dallage en pierre des zones de circulation, Tournon fait couler une chape de ciment dans laquelle sont noyées les canalisations d'un chauffage par le $\mathrm{sol}^{26}$.

La situation urbaine inédite de l'église en détermine largement le plan. Constituée de deux parcelles de terrain localisées l'une, rue Cannebière, l'autre légèrement en contrebas avenue Daumesnil entre des immeubles mitoyens, elle amène l'architecte à composer son plan selon deux axes différents habilement réunis par un narthex en forme de rotonde à sept pans (ill. 6). L'idée de cette rotonde, inspirée par la fonctionnalité anatomique du genou ${ }^{27}$, permet à l'architecte de transformer radicalement sa conception de l'intégration urbaine de l'édifice qui, de classicobaroque, devient plus "organique ", enclavée dans le tissu urbain. L'étroitesse de la parcelle située sur l'avenue Daumesnil donne à Tournon l'idée de recréer un effet de surprise absent de l'urbanisme depuis l'époque classique, en composant avec l'alignement de la façade sur les immeubles mitoyens (ill. 7). Ce choix démontre l'absence d'a priori de l'architecte à une époque, tout particulièrement au cours de la décennie, où prévaut au contraire le dégagement des monuments des constructions anciennes environnantes ${ }^{28}$. Un souci de mise en évidence dans un quartier alors dépourvu de repères monumentaux conduit Paul Tournon à souhaiter y inscrire un clocher haut de 85 mètres, phare "puissant [qui] projettera ses rayons lumineux et appellera les âmes à la source de la lumière, de la fécondité et de l'ordre " ${ }^{29}$. Son caractère imposant, finalement limité par mesure d'économie, est renforcé par la verticalité du faisceau de contreforts encadrant la loge des bénédictions. L'intégration urbaine se poursuit dans les rues adjacentes par l'alignement des façades sur celles des immeubles mitoyens. Leur austérité s'agrémente de sculptures en béton. Le pari s'annonçait difficile en raison de la discontinuité des façades. Tournon tire également parti des contraintes topographiques en exploitant le dénivelé entre les deux terrains par la création d'un escalier monumental abrité,

25. Arch. nat., 377 AP 203, f 219, 14 avril 1936 à son confrère F. Maille : "J'ai simplement passé 3 couches de Stic B sur cette dernière coupole et n'ai eu jusqu’à présent aucun ennui. Mes crédits étant extrêmement restreints, je n'ai pas pu prévoir de couverture en cuivre comme le fait notre confrère Barge pour l'église Sainte-Odile [...], mais il est évident qu'il n'y a pas mieux. " Cf. note 110 .

26. Arch. nat., 377 AP 132, f 545, 27 décembre 1934.

27. Marion Tournon-Branly, entretien, 12 décembre 2003. Cette idée sera réinterprétée quelques années plus tard à Saint-Ferdinand des Ternes par Paul Théodon, Frédéric Bertrand et Pierre Durand.

28. Que l'on songe à la mise en valeur de la cathédrale de Barcelone par Rubió i Bellver, au dégagement opéré par Prost des grandes mosquées ottomanes dans le centre ancien d'Istanbul, ou au projet ultérieur de Tournon pour la cathédrale d'Amiens.

29. Construit à la fin des années 50, il sera finalement d'une hauteur de $75 \mathrm{~m}$. Église du Saint-Esprit, Mémorial permanent..., op. cit., p. 6. 


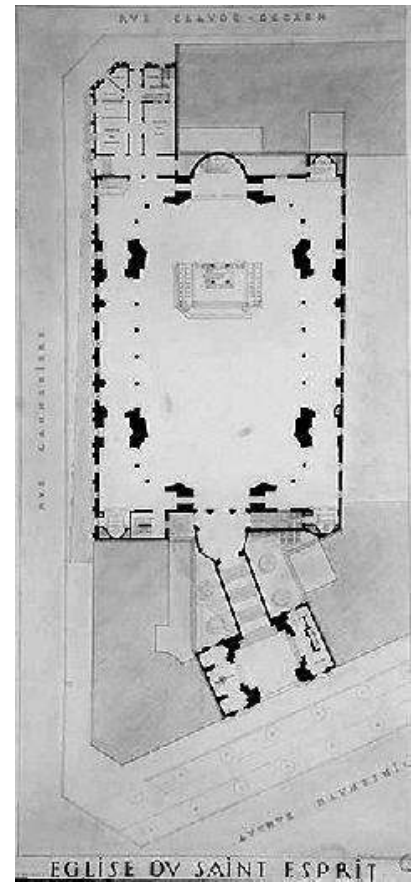

Ill. 6: Paul Tournon, Plan de l'église du Saint-Esprit, 1928, encre de Chine, mine de plomb sur papier, IFA, TOURN/D/28/1 351 AA 10/8 (photographie : Fouin Christophe () Ville de Paris, COARC, 2002. Base mémoire : DSC_0515.JPG).

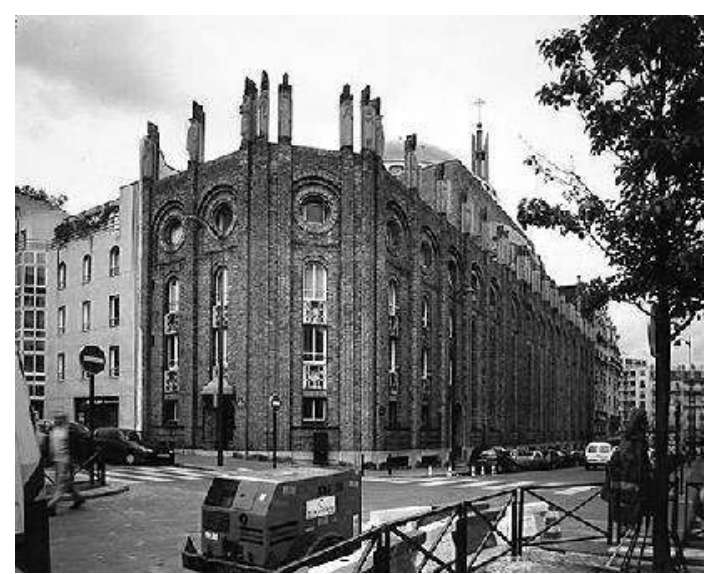

Ill. 7 : Vue des façades latérales et arrière de l'église et du presbytère du Saint-Esprit, 1976. Arch. paroissiales (photographie : Fouin Christophe (C) Ville de Paris, COARC, 2002. Base mémoire : IMG_0048.JPG). 
dont l'étroitesse rend l'ampleur de l'église haute, lieu d'un riche déploiement iconographique, d'autant plus impressionnante.

Pour l'intérieur, l'architecte se dit porté par le contenu spirituel qu'il confere immédiatement à un édifice qui sera son grand œuvre. Celui-ci s'inscrit au cœur du catholicisme dans sa vocation universelle. Depuis le porche, l'effet ascensionnel des marches, un éclairage naturel et artificiel approprié, le dépouillement croissant du décor en partie haute attirent le regard vers la coupole centrale. Ainsi l'effet architectural répond-il au sentiment mystique de l'architecte ${ }^{30}$. Tournon recycle partiellement les principales composantes du programme iconographique mis en œuvre en 1931 au Pavillon des missions catholiques à l'Exposition coloniale, où la zone supérieure présente l'Église triomphante, la zone médiane l'Église militante, la zone inférieure enfin, l'Église souffrante ${ }^{31}$ (ill. 8). Au registre supérieur, en mosaïques dites "émaux de Venise " noyées dans le béton, exécutées sur des cartons de Marcel Imbs par l'atelier Gaudin, le sommet de la coupole est occupé par une figuration de la Trinité placée dans un rayonnement lumineux diffusé en contrebas par autant de rayons de mosaïque d'or que de fenêtres. Ce mystère est transmis par les Évangélistes dont les symboles ailés, dominant chacun une scène dans laquelle se manifeste l'action de l'Esprit saint, sont figurés dans quatre médaillons situés aux pendentifs de la coupole centrale. Aux demi-coupoles, les apôtres sont regroupés sous les quatre aspects par lesquels le Christ les a désignés, en bergers, moissonneurs, vignerons et pêcheurs. L'Agneau mystique surmonte le maître autel. Au registre intermédiaire, les chapiteaux prévus en couronnement des colonnes entourant la nef, au nombre de seize, devaient représenter les grands ordres monastiques poursuivant dans le monde l'œuvre de l'Esprit saint. Sur les parements du mur d'enceinte se déploie l'action de celui-ci à travers l'histoire de l'Église, épopée évangélique commentée par des textes gravés dans le soubassement des fresques. Cette histoire se déroule depuis la Pentecôte jusqu'au XX $X^{\mathrm{e}}$ siècle. Au registre inférieur enfin, les allées devaient être revêtues de pierres « tumulaires » rappelant le prolongement de l'action de l'Esprit saint parmi les défunts. La seule finalement réalisée est un hommage à un maçon mort sur le chantier, dessinée par Tournon $^{32}$ (ill. 9). Au cour de ce dispositif, le «retable d'or» de Jean Dunand représente la vision apocalyptique de l'Agneau entouré des symboles des Évangélistes et des vingt-quatre vieillards figurés par les harpes et les coupes. La croix d'autel figure la Trinité, encadrée par la Vierge et saint Jean. Autour, tel un écrin, « la grille qui surmonte la clôture est aux emblèmes de la vigne et du blé mystique, et porte les anges que la piété des fidèles entretient autour du tabernacle » ${ }^{33}$. Il est abondamment fait recours à la symbolique chrétienne des nombres.

30. Charles Imbert, "L'église du Saint-Esprit à Paris", La Technique des Travaux, $\mathrm{n}^{\circ} 3$, mars 1933, p. 134 ; Paul Tournon, L'Église du Saint-Esprit à Paris, s.d., E.D.A.R.I.

31. Arch. nat., 377 AP 275, f 122, 10 février 1932, à Norbert Noé, secrétaire de rédaction de L'Artisan liturgique.

32. [P. Tournon], «L'église du Saint-Esprit ", Les Guides de l'Art sacré, s.d., [p. 8] ; Arch. nat., 377 AP portef. 321, f 100 et 112

33. Op. cit., [p. 8] 


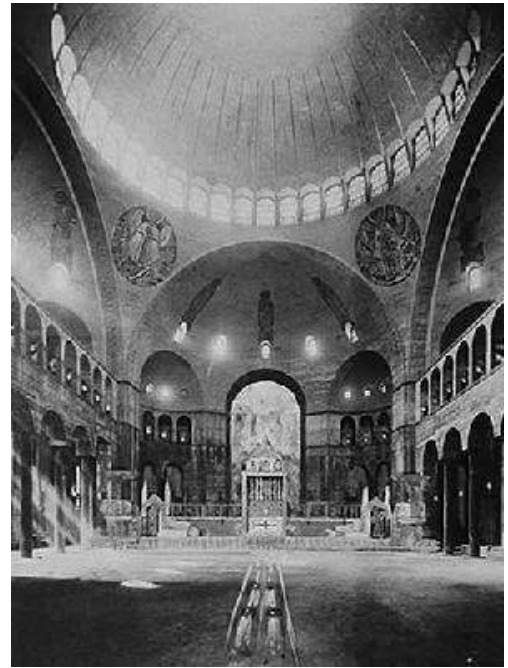

Ill. 8 : Paul Tournon, Vue intérieure de l'église du Saint-Esprit, 1932, photographie retouchée à la gouache. Arch. paroissiales (reproduction : Fouin Christophe @ Ville de Paris, COARC, 2002. Base mémoire : IMG_0069.JPG).

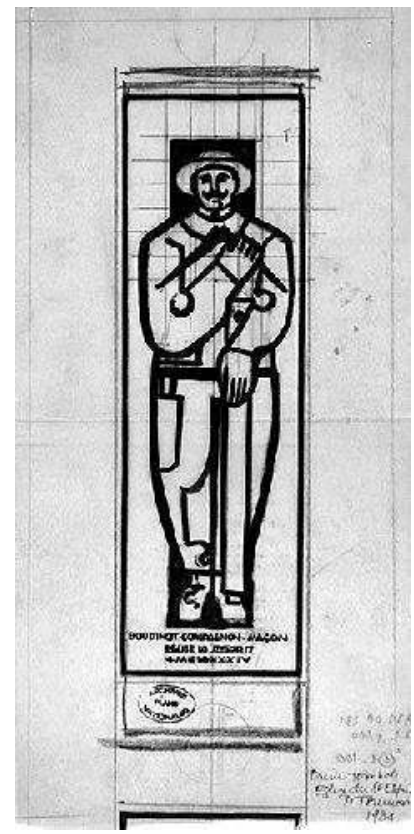

Ill. 9: Paul Tournon, Dessin d'une dalle funéraire en hommage à un maçon mort pendant le chantier, encre de Chine sur papier, 1934. Arch. nat., 377-AP/231 4 1 1-115 (photographie : Fouin Christophe (c) COARC, 2002. Base mémoire : DSC_2935.JPG) 
L'efficacité du message architectural est renforcée par la somptueuse sobriété du décor intérieur: sous le vide des coupoles se déroule un programme iconographique établi par Tournon qui, dans le vieux débat sur le rôle de l'image dans l'espace sacré, s'inscrit dans la lignée d'un Suger ${ }^{34}$. L'iconographie des sculptures extérieures rappelle celle des façades des cathédrales gothiques, avec les figures des $\operatorname{arts}^{35}$, des mois et des métiers, renouvelée par la représentation de métiers modernes, tels le chirurgien, le cameraman, le chimiste ou l'aviateur (ill. 10), le décor peint à l'intérieur est conçu comme une Bible du peuple ${ }^{36}$. Toutefois à la différence $\mathrm{du}$ Moyen Âge, c'est une histoire de l'Église chrétienne puis catholique qui se déroule depuis la Pentecôte, selon une pédagogie qui se déroule depuis le porche : le fidèle et le visiteur découvrent successivement, dans le narthex, l'histoire de La Création et les figures allégoriques des Forces matérielles et spirituelles - les facultés de l'âme et les quatre éléments (ill. 11) - fresques en camaïeu de Nicolas Untersteller (19001967) et Hélène Delaroche (dates), aux murs du baptistère Le Péché originel et son rachat par l'avènement du Christ, œuvre d'Élisabeth Branly puis, dans l'église, le Chemin de croix de Georges Desvallières (1861-1950), ainsi que la trentaine de compositions à fresque encadrant La Pentecôte, œuvre de Maurice Denis (18701943) située dans la chapelle axiale. Sept époques sont mises en correspondance avec une ou deux vertus, ou bien un don : la Pentecôte, ou la mission apostolique (sagesse, espérance) ; la semence, ou les martyrs et pères de l'Église (foi, intelligence) ; la conquête (prudence, conseil) ; l'organisation (force, justice) ; la lutte (force, science) ; l'expansion (tempérance, piété) ; la charité chrétienne, ou catholicité de l'Église (charité, crainte) ${ }^{37}$.

34. Charles Imbert, op. cit., p. 134.

35. Il ne s'agit cependant pas des arts libéraux, mais de la peinture, la sculpture, l'architecture et la musique.

36. Lettre au cardinal Verdier, 2 août 1934, Arch. nat., 377 AP 132, f 144.

37. Arch. nat., 377 AP 85, note et lettre adressées au cardinal Verdier, dactylographiées, n.d.

L'énumération des peintures qui suit mentionne les inscriptions portées par les artistes :

- à l'abside, La Pentecôte (Maurice Denis et élèves, s.d. "M. Denis 1934 »).

- $\mathrm{I}^{\mathrm{er}}$ au $\mathrm{V}^{\mathrm{e}}$ s., chapelle des martyrs (Pauline Peugniez, s.d., "Pauline Peugniez "; Henri de Maistre, s.d., "H. de Maistre 1933 "; Yvonne Soutra ; Germaine Lecler, s.d., "G. Lecler »; Lucien Weill).

- $\mathrm{VI}^{\mathrm{e}}$ au XI ${ }^{\mathrm{e}}$ s., bas-côté gauche (Élisabeth Chaplin ; Léon Toublanc; Louis Bouquet ; Raymond Weill ; Henri Marret, s.d., "H. Marret ", " 1933 » ; Marie Baranger, s.d., " Marie Baranger »).

- XII ${ }^{e}$ et XIII ${ }^{e}$ s., chapelle des confesseurs (Marguerite Hanin, s.t., "Margu. Ant. Hanin. St Bernard "; Roisin, s.t., "Roisin. Concordat de Worms "; Marie-Cécile Schmidt ; Raymond Virac, s.d., "Raymond Virac, 1933 »; Robert Génicot, s.d., « R. Génicot 33 »).

- XIV et $\mathrm{XV}^{\mathrm{e}}$ s., chapelle des vierges (catholiques des Beaux-Arts : Jean Couturat, s.d., "Couturat »; Élisabeth Faure, s.d. "É. Faure, 1935 »; Marthe Flandrin ; Émile Beaume, s.d., "Émile Beaume Pinxit »; Mériel-Bussy, s.d., "A. Mériel Bussy Pinxit 1933 »; Odette Pauvert, s.d., "Odette Pauvert ").

- XVI e au XVIII s., bas-côté droit (Robert Pougheon, Jean Dupas et Ovile, s.d., "Jean Dupas, $1934 »)$.

- XIX et $\mathrm{XX}^{\mathrm{e}}$ s., chapelle du travail (Eugène Chapleau, s.d., "[?] Chapleau, 193[?] "; André et Yvanna Lemaître, s.d., "André-Hubert Lemaitre "; Sylvaine Collin, s.d., "Sylvaine Collin, 1933 "; Henri Charlier ; Annette Storez, s.d., "A. Storez, 1934 »; Valentine Reyre).

- Dans le bas-côté gauche figure en outre un panneau peint sur plâtre, Suvre de Louise Lenoir, une Mise au tombeau présentée à l'exposition d'art religieux de l'Hôtel des ducs de Rohan en 1934. 


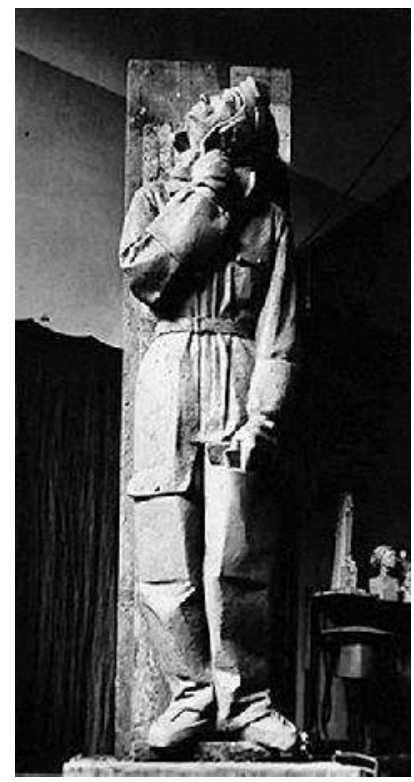

Ill. 10 : Roger Prat, L'Aviateur, argile, modèle original pour une statue en béton moulé, 1943. Photographie anonyme, IFA, TOURN/A/6 351 AA 2/1 (reproduction : Fouin Christophe () Ville de Paris, COARC, 2002. Base mémoire : DSC_0577.JPG).

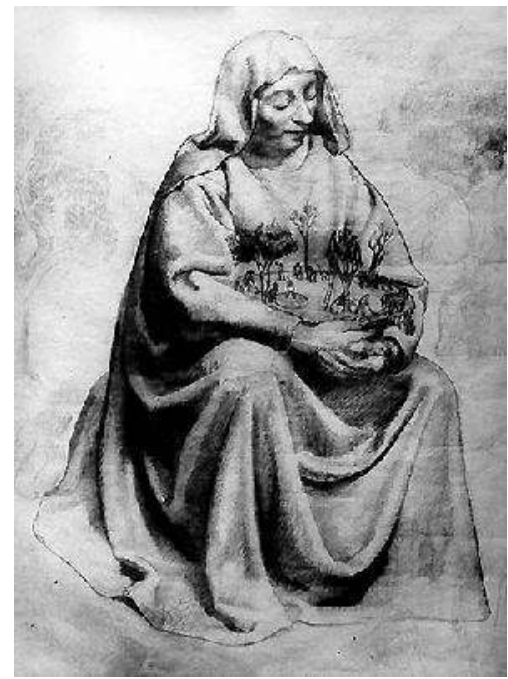

Ill. 11 : Nicolas Untersteller, La Terre, étude pour le décor du narthex de l'église du Saint-Esprit, [1933], fusain sur papier calque, musée Carnavalet (photographie : Fouin Christophe () Ville de Paris, COARC, 2004. Base mémoire: DSC_3034.JPG). 
Pourtant, nombre d'éléments initialement prévus ne furent jamais réalisés, faute d'argent. Deux grandes statues de béton, figurant saint Pierre et saint Paul, devaient encadrer la façade principale. À l'intérieur de l'église, des chapiteaux devaient être sculptés "en taille directe dans un matériau ayant la consistance de la pierre tendre " 38 (ill. 12), un bas-relief était prévu pour orner la tribune du grand orgue, la sculpture en incrustation en noir sur blanc des dalles en pierre de Larrys devait scander au sol les zones de circulation (ill. 9). Tournon envisageait en outre un décor plus riche et coloré pour le ciborium dont les arcatures du couronnement, traitées à jour dans l'esprit de celles de l'église, devaient porter des figures d'anges en basrelief ou en émail ${ }^{39}$. Par mesure d'économie encore, en raison de la présence alors d'une construction obstruant la lumière le long de cette façade, les vitraux ne furent jamais commandés pour le collatéral nord-ouest. Celui-ci resta éclairé par de la vitrerie industrielle incolore rehaussée de couleurs à froid ${ }^{40}$. Sur les piliers, la pierre dure et le marbre devaient déployer "leurs beaux effets naturels " 41 . Tournon avait une vision initiale riche et colorée, ce qui nous oblige à réviser la perception brutaliste que l'on a aujourd'hui de l'ensemble. Il ne faut toutefois pas écarter cette dernière dimension, revendiquée à plusieurs reprises par l'architecte ${ }^{42}$ : l'hypothèse d'un revêtement en pierre jusqu'à la corniche laissait la part belle au déploiement du béton brut dans les parties les plus élevées (ill. 13). La coupole centrale, pourtant solidement ancrée dans d'énormes piliers rappelant les points cardinaux, s'assimile à la voûte céleste. Tout cela modifie notre appréciation de l'ensemble, sans pour autant la transformer radicalement. Tournon explique le manque de visibilité des peintures, une certaine ambiguïté dans leur mise en valeur, par la volonté d'attirer le regard vers la grande coupole centrale ${ }^{43}$. Ainsi la posture de l'architecte, qui privilégie l'effet spatial, est-elle mise en tension avec une sensibilité plus picturale.

38. Arch. nat., 377 AP 275, f 91, 4 février 1932, lettre à Mgr Crépin ; Arch. nat., 377 AP 85, liasse de croquis et photographies : au recto figure le croquis d'une colonne avec un chapiteau en médaillon; au verso, une liste avec, en colonne de gauche, les ordres religieux et, en colonne de droite, des noms de sculpteurs (de Villiers, incorrectement orthographié Devillers, Martin, Dubois, Roux, Parvillée, Dufrasne, Nicot, ainsi que trois noms illisibles); puis, un croquis localisant la représentation des ordres religieux et celle des artistes. C 85, dossier T, 19 mai 1932 : H $110 \mathrm{~cm}$, " en taille directe dans un matériau ayant la consistance de la pierre tendre ". Sur la face tournée vers la nef, "la figure caractéristique d'un saint ou d'un frère ou père de cet ordre. Sur les trois autres faces pourraient être les attributs ou des scènes intéressant l'ordre envisagé. Sur une bande au-dessous serait une inscription ".

39. Arch. nat., 377 AP 202, f 73, 24 juillet 1934, lettre à Chéret.

40. Ayant viré ou disparu depuis, ce qui crée une luminosité d'autant plus agressive dans l'atmosphère filtrée de l'église, que l'immeuble fut démoli en 1962 : Arch. nat., 377 AP 85, liasse "Église ", 26 septembre 1962, lettre du P. Delouvrier à Paul Tournon.

41. La Technique des travaux, $\mathrm{n}^{\circ} 3$, mars 1933, p. 134. "Tous les piliers en béton armé sont appelés à recevoir un revêtement de pierre dure ou de marbre ", avait annoncé Paul Tournon antérieurement : "L'Architecture des églises et l'église du Saint-Esprit ", Le Messager du Saint-Esprit, [2 ${ }^{\mathrm{e}}$ semestre 1938], p. 13 (conférence prononcée le 9 juillet 1930).

42. Op. cit., et Arch. nat., 377 AP 173, f 397, 4 janvier 1930 : «Le forfait de ciment armé de cette église, qui restera brute de décoffrage, est de 2000000 francs. ”

43. Arch. nat., 377 AP 201, f 73, 31 janvier 1935, au P. Roulin. 


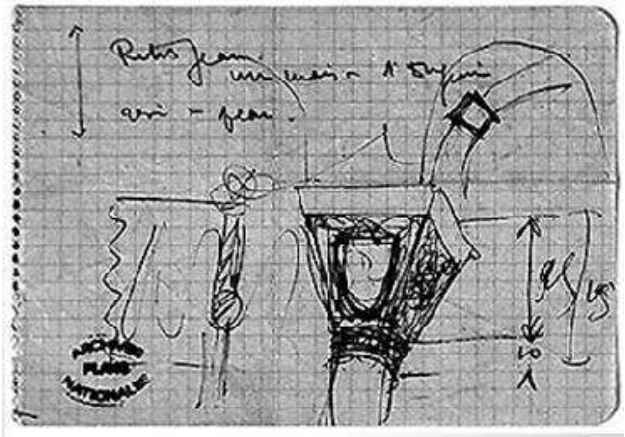

Ill. 12 : Paul Tournon, Croquis pour un chapiteau de l'église du Saint-Esprit, 1934, mine de plomb. Arch. nat., 377-AP/231 41 2-186 (photographie : Fouin Christophe (C) Ville de Paris, COARC, 2002. Base mémoire : DSC_2919.JPG).

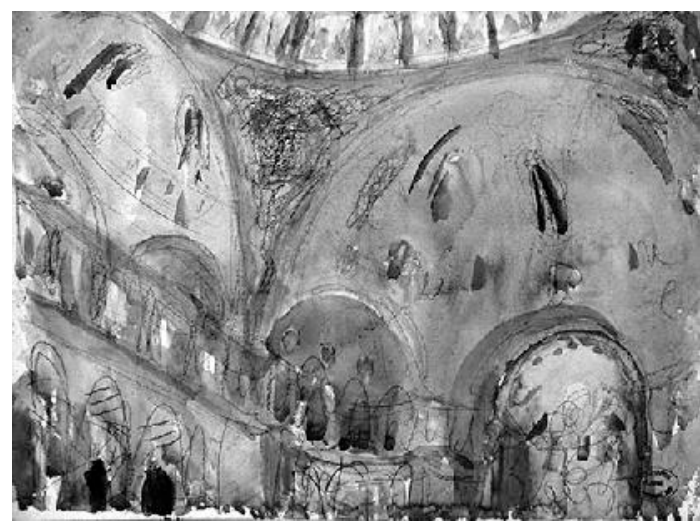

Ill. 13: Paul Tournon, Étude de couleurs pour l'intérieur de l'église du Saint-Esprit, vers 1932, mine de plomb et aquarelle. Arch. nat., 377-AP/266 (photographie : Fouin Christophe (C) Ville de Paris, COARC, 2002. Base mémoire : DSC_2972.JPG).

Au cours de la première réunion de la commission diocésaine d'architecture du programme des églises à construire dans la périphérie de Paris - vraisemblablement en janvier 1932 -, le cardinal recommande aux architectes de "réserver de grandes surfaces destinées à recevoir une décoration peinte ou sculptée afin de donner du travail aux artistes particulièrement éprouvés en ces temps de crise " ${ }^{44}$. Dès l'origine, Tournon fait appel à quelques personnalités - Maurice Denis, Georges Desvallières, Jean Dupas - et à certains groupements corporatifs - les Ateliers d'art

44. Arch. nat., 377 AP 275, f 272, 21 mars 1932, compte-rendu au directeur des Chantiers du cardinal. L'organisation du chantier de peinture murale peut être reconstituée grâce au dépouillement de la correspondance, auquel on peut ajouter utilement un dossier établi par Caroline Piel (D.R.A.C. d'Île-de-France), ainsi qu'un entretien avec Marion Tournon-Branly, 12 décembre 2003 et une correspondance avec Louis-Paul Untersteller, 31 août 2004 (Arch. COARC). 
sacré, les Ateliers de la fresque, les Artisans de l'autel, L'Arche, auxquels viennent s'adjoindre quelques Catholiques des Beaux-Arts -, reprenant la méthode inaugurée pour le décor du Pavillon des missions. La commande et la réalisation des peintures s'échelonnent du printemps 1932 à 1935. Fort d'une "vérité fondamentale" - « on ne compose pas de la même manière une surface peinte à l'huile, une tapisserie, une mosaïque ou une fresque »-, Tournon entend contribuer au renouveau de la peinture murale ${ }^{45}$. Soucieux de l'unité d'un ensemble confié à près de quarante artistes, l'architecte donne pour consigne de peindre à fresque, de conférer la même hauteur aux figures en employant les mêmes colorations pour les fonds, le rouge devant être la tonalité dominante.

L'importance accordée à la lumière se révèle dans la correspondance relative aux vitraux, confiés dans leur quasi-intégralité à Louis Barillet (1880-1948), hormis ceux des bas-côtés constitués de verre cathédrale opale blanc à l'intérieur, doublé de verre coloré transparent à l'extérieur, le tout maintenu dans des plombs et claustras en béton ${ }^{46}$. Pour la base de la grande coupole, ils sont "garnis de verres antiques colorés, très transparents dans les verrières hautes de l'édifice et de plus en plus opaques à mesure qu'on se rapproche du sol. Ces verrières ont été volontairement réduites à un jeu de fonds pour laisser toute leur valeur aux fresques ${ }^{47}$. Tournon privilégie l'effet ascensionnel, en second lieu les fresques, conférant aux vitraux le rôle d'un faire-valoir discret. L'éclairage artificiel appuie cette conception : l'architecte développe au Saint-Esprit le principe, inauguré au Pavillon des missions, de l'illumination des voûtes par "un éclairage indirect mettant en valeur la mosaïque des coupoles et les fresques du pourtour. Il est réalisé par le moyen de projecteurs posés au-dessus des portiques latéraux [au-dessus des corniches] et les sources de lumière sont absolument invisibles $"{ }^{48}$. Ce dispositif d'ambiance devait être complété d'un système traditionnel de couronnes de lumières accrochées sous la galerie, entre les colonnes ${ }^{49}$.

Les grandes figures des apôtres sur les coupoles sont « réalisées en mosaïques et coulées en même temps que les coupoles; les cubes de mosäque montés sur papier ayant été posés directement sur les coffrages avant la coulée des coupoles ", procédé permettant d'économiser du temps de main-d'œuvre et un échafaudage spécial ${ }^{50}$.

45. Arch. nat., 377 AP 275, f 369, 22 avril 1932, lettre à Beaume.

46. Exceptions : ceux de la crypte, sur des cartons de Marcel Imbs (1882-1935), furent exécutés en 1928 par Paul Louzier ; les deux éclairant l'autel du Sacré-Cœur, au décor confié à des artistes des Ateliers d'art sacré sous la direction de Henri de Maistre, furent exécutés par Jean Hébert-Stevens.

47. Arch. nat., 377 AP 276, d 345, 3 octobre 1932, au P. Hattais : Tournon et Maurice Denis viendront voir avec Louis Barillet les vitraux de l'église; Arch. nat., 377 AP 207, d 547, 9 juin 1934, à Henri Marret (1878-1964).

48. Arch. nat., 377 AP 201, f 73, au P. Roulin, 31 janvier 1935. À l'instar de l'éclairage intérieur de l'église Saint-Jean-Bosco, dans le $\mathrm{XX}^{\mathrm{e}}$ arrondissement de Paris (D. et René Rotter, architectes). Pour autant, Tournon ne prône pas systématiquement l'éclairage des voûtes.

49. Arch. nat., 377 AP portef. 231, f 194.

50. Arch. nat., 377 AP 201, f 73, 31 janvier 1935, au P. Roulin ; Arch. nat., 377 AP 199, f 567, 28 novembre 1931 ; Arch. nat., 377 AP 199, f 164, 7 juillet 1931, à Gaudin ; Paul Tournon, Le Message du Saint-Esprit, p. 12, conférence du 9 juillet 1930. 
Parallèlement au transfert de la cuve baptismale depuis la crypte vers la chapelle des fonts, le reste du décor intérieur se poursuit au printemps 1935 : revêtement et gravure des textes du soubassement des fresques, marouflage sur le ciment du Chemin de croix de Georges Desvallières (1861-1950), mise en place des confessionnaux dessinés par l'architecte, des bénitiers en aluminium martelé de Richard Desvallières (1893-1962), du retable de la chapelle de la Vierge en bronze doré et argenté, dessiné Jean Dunand (1877-1942) et exécuté par Raymond Subes (18931970) ${ }^{51}$. La structure du chœur est revêtue de marbres de diverses teintes. La grille de la clôture, également dessinée par l'architecte, est exécutée par Jules Chéret (1836-1932) en 1938 (ill. 14). La porte de l'église, en fer doré à la feuille, est commandée en $1931^{52}$. Suivent la commande de l'abat-voix de l'ambon des Évangiles à Roger Prat, celle des retables des autels occupant les chapelles d'angle à Roger de Villiers et Jacques Martin ${ }^{53}$. Les sculptures extérieures demandées à Sarrabezolles, les "Quatre Arts» (La Peinture, La Sculpture, L'Architecture et La Musique) situés dans l'axe de la grande coupole côté rue Cannebière, avaient été exécutées en 1932, tandis que la réalisation des autres sculptures pour les façades arrière et latérale attend 1942 et 1943 (ill. 10), et les symboles des Évangélistes couronnant le clocher, la construction de celui-ci à la fin des années 1950 (ill. 15). Les douze bas-reliefs en ciment moulé insérés en partie basse de la façade rue Cannebière, figurant les Mois, sont exécutés en 1941 par Feuerstein, Gibert, Guignier et Munzinger. Les Métiers sont l'œuvre de Jan et Joël Martel, Émile Morlaix, Jacques Martin, Marcel Homs, Lucien Fenaux, Pierre Thézé, Georges Serraz, René Leleu, Gabriel Dufrasne, François Bazin, Roger Prat ${ }^{54}$ (ill. 11).

Le montant de l'enveloppe allouée à la réalisation des fresques et mosaïques est de 364000 francs pour $825 \mathrm{~m}^{2}$, soit moins de 450 francs le $\mathrm{m}^{2}$, somme globale mise à la disposition de l'architecte par le cardinal, dans laquelle une rémunération de 400 francs au $\mathrm{m}^{2}$ est à répartir par forfaits entre les artistes, à l'exception de Dupas, Desvallières et Denis, "qui sont leurs maîtres " et du couple Untersteller, qui ne demande aucune rétribution ${ }^{55}$. La somme est nette pour l'artiste, le prix

51. Arch. nat., 377 AP 201, f 169, 4 mars 1935, lettre à Sallé. L'autel fut supprimé lors du réaménagement de la chapelle dans les années 1970 (Marion Tournon-Branly).

52. Dernier élément de décor à avoir été réalisé, la grille encadrant la porte vitrée du porche, aux flammes en métal doré, qui signe en 1960 l'achèvement de tout le massif d'entrée.

53. Le Sacré Cœur, bas-relief en pierre calcaire, commande en 1939, exécution en 1941 : Arch. nat., 377 AP portef. 231, n $13-94$; Arch. nat., 377 AP 85, dossier T, 27 décembre 1943. La mort de saint Joseph, haut relief en pierre calcaire, s.d.b.d., "Jacques Martin, 1946 " (Arch. nat., 377 AP 85) ; saint Jean Eudes, bas-relief en pierre calcaire, s.d.b.mil., "Jacques Martin, 1952 ", sur une proposition de Paul Tournon (Arch. nat., 377 AP portef. 231, f 25, 1951).

54. Pierre Ladoué, "L'art vivant: les achats et commandes de l'État aux artistes en 1941 ", Revue des Beaux-Arts de France, no 1, octobre-novembre 1942, p. 23-30 ; "L'art religieux et la décoration des églises de 1940 à 1945 ", Les Études, n 1 , janvier à mars 1946, t. 218, p. 112-123. Arch. nat., 377 AP 85, 189 et 198. Article en préparation.

55. Sur la collaboration avec Nicolas Untersteller et Hélène Delaroche, cf. un courrier de Louis-Paul Untersteller, en date du 31 août 2004 (COARC) : les peintres auraient tenu à contribuer au décor de l'église du Saint-Esprit, même à titre gracieux, ce qui laisse supposer qu'ils ne participèrent pas $\mathrm{au}$ " concours " destiné à sélectionner les artistes. 


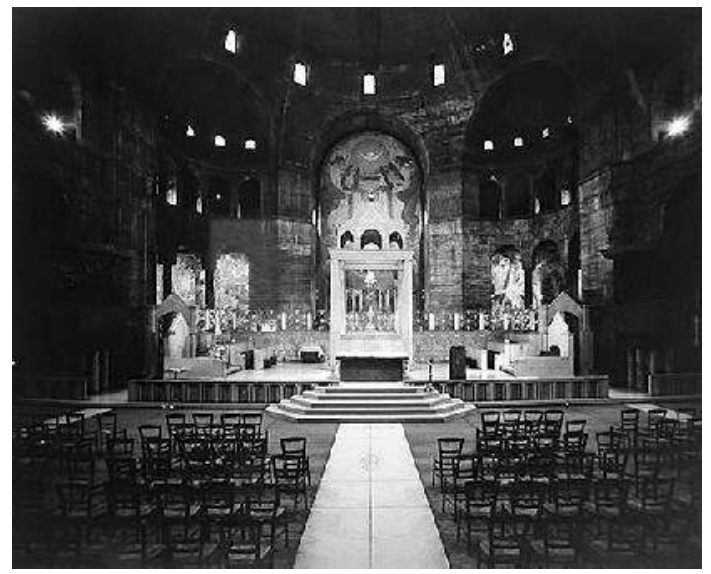

Ill. 14: Vue du chour de l'église du Saint-Esprit. Photographie: COARC (photographie: Fouin Christophe (C) Ville de Paris, COARC, 2001. Base mémoire : IMG_0042.JPG).

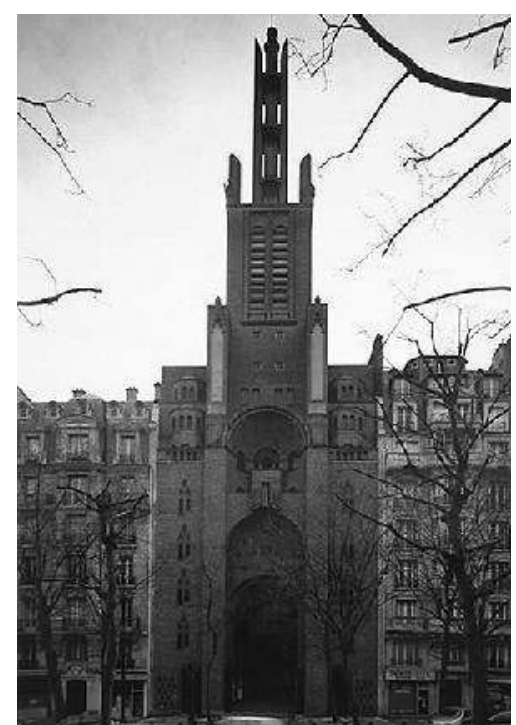

Ill. 15: Vue de la façade sur l'avenue Daumesnil de l'église du Saint-Esprit. Photographie: COARC (photographie : Fouin, Christophe @ Ville de Paris, COARC, 2001. Base mémoire : IMG_0063.JPG).

des échafaudages et enduits étant supporté par l'archevêché. Maurice Denis se voit attribuer un forfait de 70000 francs pour la peinture de l'abside, ce qui correspond à une rémunération de 500 francs $/ \mathrm{m}^{2}$, et Desvallières, un forfait de 20000 francs. Aucune indication ne figure dans les archives sur celle de Dupas. Quant aux mosaïques, elles ont coûté 2450 francs le $\mathrm{m}^{2}$ posé, comprenant le forfait à Imbs et l'exécution aux voûtes par la maison Gaudin en 1931, à raison de 500 francs le $\mathrm{m}^{2}$ pour les figures comportant de l'or et de l'argent, et 200 francs le $\mathrm{m}^{2}$ pour 
les inscriptions. Moyennant quelques compléments ainsi que le coût des mosaïques et des vitraux hauts, le total s'élève à un peu plus de 400000 francs.

Bien que le prêtre officiant tourne le dos aux fidèles alors que d'autres églises ont adopté l'autel à la romaine, Tournon fait siennes la plupart des nouvelles recommandations émanant du mouvement liturgique, faisant de l'église un lieu de grande visibilité, où l'audition est facilitée par la présence de haut parleurs, et un lieu confortable grâce à un système de chauffage par le sol répartissant la chaleur de manière régulière ${ }^{56}$. La dimension utilitaire de ce confort exalte l' "américanisme " de sa conception moderne. Un quotidien généraliste emploie le terme de building à propos du clocher tel qu'il est projeté en $1933^{57}$. L'ambition du maître d'œuvre est relayée par l'archevêché de Paris, qui fait de ce chantier une vitrine de sa politique constructive, au point de demander à Tournon un rapport préconisant un pourcentage du budget de construction à réserver à la commande d'œuvres d'art originales : lors de la première réunion de la commission diocésaine d'architecture pour l'étude du programme des églises à construire dans la périphérie de Paris, la somme prévue pour les décorations peintes et sculptées et les mosaïques est évaluée à $11 \%$ de la dépense totale de la construction de l'église du Saint-Esprit ${ }^{58}$. Qualifiée de "reine des chantiers" par Mgr Verdier, l'édification de cette église est, jusqu'en 1935, la grande affaire des Chantiers du Cardinal ${ }^{59}$ et considérée comme suffisamment importante pour que la Ville de Paris accepte de relayer l'association diocésaine dans le financement de son achèvement ${ }^{60}$. En affichant l'ambition d'en faire un modèle, Paul Tournon se pose en acteur majeur du renouveau de l'art

56. Arch. nat., 377 AP 202, f 406, 30 octobre 1934, lettre à Jean Favier. Panneaux chauffants système Crittall breveté SGDG, posés dans le sol sous le dallage de l'église, "béton additionnel constituant l'enrobement des canalisations de chauffage; revêtu d'une chape au mortier de ciment lissée et bouchardée au rouleau, de 2 c; la surcharge libre sur ce plancher sera de $200 \mathrm{~kg} / \mathrm{m}^{2}$ " : Arch. nat., 377 AP 132, f 545, 27 décembre 1934; L'Art sacré, no 7, janvier 1936, p. 17. Saint-Pierre de Chaillot est équipé du même système.

57. Coupure de presse non identifiée, Arch. paroissiales; Le Jour, 30 octobre 1933, Tournon à Antoine de Courson, Arch. paroissiales.

58. Arch. nat., 377 AP 275, f 170, 24 février 1932, rapport au cardinal Verdier ; Arch. nat., 377 AP 272, 21 mars 1932, note au vicaire général Touzé. La réalité sera nettement moins favorable aux arts plastiques.

59. Pour comparer avec des édifices de proportions similaires, le prix de revient de Saint-Pierre de Chaillot et de Sainte-Odile, vraisemblablement plus élevé (on ne dispose pas de données chiffrées globales) est assumé, dans ces paroisses riches, par un financement in partibus, grâce notamment au dynamisme inlassable de Pierre l'Ermite, chargé par le cardinal de la construction de Sainte-Odile.

60. Pour comparer avec des édifices de proportions similaires, le prix de revient de Saint-Pierre de Chaillot et de Sainte-Odile, vraisemblablement plus élevé (on ne dispose pas de données chiffrées globales) est assumé, dans ces paroisses riches, par un financement in partibus, grâce notamment au dynamisme inlassable de Pierre l'Ermite, chargé par le cardinal de la construction de SainteOdile. Arch. nat., 377 AP 85, 12 mars 1942, à Charles Venner : complément d'estimation demandé par Venner à Tournon à partir de mars 1941, et cession de propriété à la Ville de Paris qui s'en est suivie ; cela tandis que certaines églises construites sur des terrains appartenant à la commune resteront inachevées. Cf. Saint-Gabriel $\left(20^{\mathrm{e}}\right)$, Sainte-Hélène $\left(18^{\mathrm{e}}\right)$. Miriam Simon, "La construction de lieux de culte sur les anciens bastions de. Paris dans l'entre-deux-guerres ", Revue d'Histoire de l'Église de France, t. 90, nº 225, 2004, p. 453-479. 
religieux dans l'Entre-deux-guerres et est accepté comme tel par la presse spécialisée modérée ${ }^{61}$.

\section{Une ouvre complète}

L'organisation du chantier fait montre d'une maîtrise d'œuvre dirigiste qui a pour corollaire une conception totalisante de l'œuvre architecturale à l'instar, au milieu du XIX ${ }^{e}$ siècle, de Boileau à Saint-Eugène-Sainte-Cécile, Lusson à SaintJean-Baptiste de Belleville, Ballu à la Sainte-Trinité, Baltard à Saint-Augustin, qui impriment une homogénéité complète au décor intérieur de ces édifices. Elle rappelle aussi le Baltard responsable des travaux dans les églises parisiennes, se réclamant de l'esprit des corporations du Moyen Âge pour la redécoration de SaintGermain-des-Prés. Tout en faisant appel à des groupements contemporains d'art sacré, l'architecte est seul responsable du programme décoratif et iconographique de l'ensemble. Convaincu que «la prééminence de l'architecture française est subordonnée à l'ordonnance des trois arts " ${ }^{62}$, reprenant à son compte la synthèse des arts en vogue dans certains milieux artistiques depuis le milieu du XIX ${ }^{c}$ siècle, Tournon a l'opportunité de mettre sa théorie en application. Considérant que l'éducation des artistes en matière de décoration monumentale a été négligée depuis trop longtemps, il crée aux Beaux-Arts un concours des Trois Arts et fait aménager un atelier d'art monumental permettant aux sculpteurs et aux peintres de travailler ensemble sur des projets, avec des architectes ${ }^{63}$. L'utilisation du béton se développe dans ce contexte de soumission des artistes à l'architecture, observable dans les nombreux tympans sculptés et décors tapissants couvrant les parements de nombreux édifices publics, que ce soit dans la pierre ou en peinture, avec la vogue de la fresque. La conception muraliste du décor par Tournon s'exprime pleinement ici. S'agissant d'art religieux, il a la satisfaction de bénéficier de la disposition bienveillante des évêques.

Exemple remarquable d'une soumission des artistes à l'architecture, une collaboration régulière s'est instaurée entre Tournon et Sarrabezolles pour des édifices religieux de la région parisienne, à l'instar d'autres partenariats entre architecte et

61. Le Journal, 7 octobre 1933; L'Architecture, 15 octobre 1934, p. 355-372 ; "L'Église du SaintEsprit à Paris ", La Technique des Travaux, nº 3, mars 1933, p. 130-144.

62. Arch. nat., 377 AP 202, f 291, 21 septembre 1934, à Pol Gosset.

63. Nicolas Untersteller, successeur de Paul Tournon à la tête de l'École des Beaux-Arts, dotera fortement ce concours et le renommera Concours d'art monumental : 31 août 2004, lettre de LouisPaul Untersteller, documentation de la COARC. Arch. nat., 377 AP 205, f 578, 12 mars 1946; Arch. nat., 377 AP 214, f 607, 29 octobre 1943, notice pour l'Académie: Tournon est nommé directeur de l'École des Beaux-Arts et de l'École des Arts décoratifs par Louis Hautecœur, secrétaire général des Beaux-Arts, qui veut un architecte à la tête de ces écoles, en raison de son engagement en faveur des trois arts. Cf. aussi "L'interdépendance des trois arts dans l'œuvre d'architecture ", Bulletin de la classe des Beaux-Arts de l'Académie royale de Belgique, t. XVIII, 1961. 
sculpteur ${ }^{64}$. Une grande amitié unit ce tandem qui s'était constitué en 1926 avec le chantier de Villemomble (ill. 3). Sarrabezolles, inventeur de la taille directe dans le béton en prise, réserve quasiment cette technique à l'art religieux à partir de cette date, créant un style propre lié à l'obligation de franchise et de simplicité ${ }^{65}$. À l'origine de cette invention, contraint par l'urgence à l'occasion de l'Exposition des arts décoratifs de 1925, l'artiste recourut à du plâtre frais pour sculpter deux panneaux destinés au palais de l'Afrique occidentale française. C'est en transposant cette méthode que les deux amis trouvèrent comment réaliser le programme de Villemomble ${ }^{66}$. Ils œuvrèrent parallèlement ensemble à un projet de chapelle de pèlerinage en forme de statue dédiée à saint Christophe, destinée aux automobilistes, suivant en cela la vogue des statues géantes inaugurée au XIX ${ }^{\mathrm{e}}$ siècle et poursuivie au $\mathrm{XX}^{\mathrm{e}}$ siècle grâce aux facilités apportées par le recours au béton armé ${ }^{67}$. S'agissant du Saint-Esprit, leur collaboration se déroule en plusieurs étapes. Pour la crypte, en 1928, le décor des ambons (aigle et colombe), ainsi que les statues de Saint-Joseph et Notre-Dame de la Paix pour les autels latéraux; en 1932, sur les contreforts centraux de la façade rue Cannebière ${ }^{68}$; au clocher, à la fin des années 50, les symboles des Évangélistes selon la vision d'Ézéchiel (ill. 15). Tournon avait en outre envisagé d'orner l'encadrement de la porte de la rue Cannebière ${ }^{69}$. Cette fructueuse collaboration s'illustre en 1928, à Sainte-Thérèse d'Élisabethville, dont la façade est ornée en haut relief de trente cinq figures allégoriques et de saints exécutées en six semaines (ill. 4). En 1931, Sarrabezolles sculpte Les Quatre races humaines, hautes de sept mètres, au clocher du Pavillon des missions (reconstruit en dur en 1932-1933 à Épinay-sur-Seine (ill. 6). En 1934 enfin, c'est une statue

64. Couvègnes avec Duval et Gonse en Picardie, Vigoureux et les frères Braemer avec Besnard à Paris (Saint-Christophe de Javel). Dans ces cas cependant, la finesse du grain est recherchée, corollaire d'un refus de tirer un parti expressif de la texture et de la mise en œuvre du matériau.

65. Geneviève Appert-Sarrabezolles, Marie-Odile Lelièvre, Carlo Sarrabezolles, sculpteur et statuaire, 1888-1971, Paris, Somogy, 2003.

66. Sarrabezolles proposa d'utiliser un béton non encore complètement pris et effectua des essais sur le chantier, avec un mélange de 350 de ciment pour 1000 de gravier et de sable. 12 heures de prise rendaient la sculpture possible, sans que le bloc soit encore trop dur. Il fit exécuter des coffrages sur 2,50 $\mathrm{m}$ de hauteur de personnage, en commençant par le bas, à raison de huit jours pour faire un tour complet, en suivant l'équipe des coffreurs et des couleurs, en continuant au niveau supérieur, et ainsi de suite jusqu'à la base des séraphins, exécutés antérieurement. Il reprit ensuite les parties déjà faites dans une matière désormais sèche et très dure, accusant certains traits, fixant quelques expressions et colorations, gravant les inscriptions. Commencé le 20 août, le chantier s'acheva le 3 novembre, soit une durée de 63 jours. Cette façon de travailler constitue un tour de force, car le sculpteur n'avait pas de points de repère et devait travailler sans recul possible en évitant les différences d'échelle d'un niveau à l'autre, le tout dans des conditions de grande difficulté technique. Les figures sont hiératiques et simples ; quelques visages furent sculptés à la ressemblance de ceux qui contribuèrent à l'érection du clocher : Carlo Sarrabezolles, «La sculpture sans maquette par taille directe du béton en prise ", Bulletin de la Société d'encouragement pour l'industrie nationale, octobre 1933, p. 529-534.

67. Chapelle Saint-Christophe au Jajolet, 1926-1927, projet non réalisé.

68. Arch. nat., 377 AP 275, Tournon à Sarrabezolles, le 11 mars 1932.

69. Document non daté, archives Sarrabezolles. 
monumentale de Saint Pierre apôtre, haute de quatorze mètres, qui est exécutée pour une chapelle de secours située dans le quartier de l'île-Saint-Pierre, à Alfortville ${ }^{70}$.

Concernant les peintures murales du Saint-Esprit, il est écrit partout que les artistes furent sélectionnés par concours, ce qui est partiellement exact, l'architecte se servant de l'avis de confrères réunis à son agence pour avaliser son choix des peintres. Élisabeth Branly, Maurice Denis, Jean Dupas et Georges Desvallières furent sollicités d'emblée. Le couple Untersteller, qui devait initialement travailler avec Jean Dupas puis Marthe Flandrin, se vit finalement confier, seul, le décor du narthex ${ }^{71}$. S'agissant des autres, les chefs d'équipe proposèrent des noms au sein du groupement d'artistes auquel ils appartenaient ${ }^{72}$. Les seize chapiteaux devaient également être répartis entre des sculpteurs appartenant à ces mêmes groupes ${ }^{73}$. Paul Tournon se livre à un important travail d'organisation et de coordination. L'architecte avait expérimenté sa méthode de travail à la faveur de la construction du Pavillon des missions, mais le chantier du Saint-Esprit est conçu différemment en raison de l'unité du volume intérieur et de l'appel à un plus grand nombre d'artistes, tandis que lui-même concentre la maîtrise d'œuvre dans sa seule agence. Au Pavillon pontifical, en 1937, il désignera à nouveau plusieurs jeunes architectes, responsables chacun d'un secteur, pour l'exécution des détails de ce grand ensemble.

À l'instar de ses relations avec les membres du clergé, celles avec les artistes sont toujours respectueuses, mais non dépourvues de fermeté lorsque la cohérence du décor l'exige. À Maurice Denis, Tournon redit « la nécessité d'une couleur dominante donnant une tache simple dans le haut de [sa] composition, corollaire de celle de donner "une coloration unitaire" à l'abside " ${ }^{74}$. À Henri Charlier (18831975) il écrit, le 13 mai 1935 : "Il y a toujours, vous le savez, une chose qui me chagrine ce sont vos auréoles losanges ou carrées qui ont bien, en effet, été pratiquées par les Byzantins mais pour attirer l'attention sur le personnage d'importance tout en montrant qu'il n'était pas saint, l'auréole circulaire étant réservée à ceuxci. On m'en a fait d'ailleurs déjà de nombreuses fois la réflexion " ${ }^{75}$. Le décor de chacune des "chapelles " est à un groupement distinct, bénéficiant de l'habitude de travail en commun de ces structures pour des compositions à la fois unitaires et fragmentées ${ }^{76}$ : les Ateliers d'art sacré pour la première, sous la direction de Henri de Maistre; les ateliers de La Fresque pour la deuxième, sous la direction de Henri Marret (ill. 16). L'amitié entre Tournon et son confrère Maurice Storez (1875-1959), fondateur de L'Arche, vaut à ce groupement de se voir confier le

70. Arch. nat., 377 AP 207, 18 décembre 1933 à Outardel : restée inachevée en 1934 par manque de crédits, cette chapelle servait de piédestal à une statue monumentale qui fut détruite et remplacée dans les années 80 par l'actuel clocher.

71. Arch. nat., 377 AP 275, f 547, 11 juin 1932.

72. Arch. nat., 377 AP 276, 20 juillet 1932, à M. Vieulle.

73. Rapport à Verdier, 24 février 1932. Arch. nat., 377 AP 85, liasse "croquis/photographies ».

74. Arch. nat., 377 AP 207, f 333, 3 avril 1934 ; Arch. nat., 377 AP 207, f 468, 16 mai 1934. Arch. nat., 377 AP non coté, 28 juillet 1934, M. Denis à P. Tournon.

75. Arch. nat., 377 AP 201, f 353, 13 mai 1935.

76. Arch. nat., 377 AP 275, f 432, 12 mai 1932, à Marthe Flandrin. 


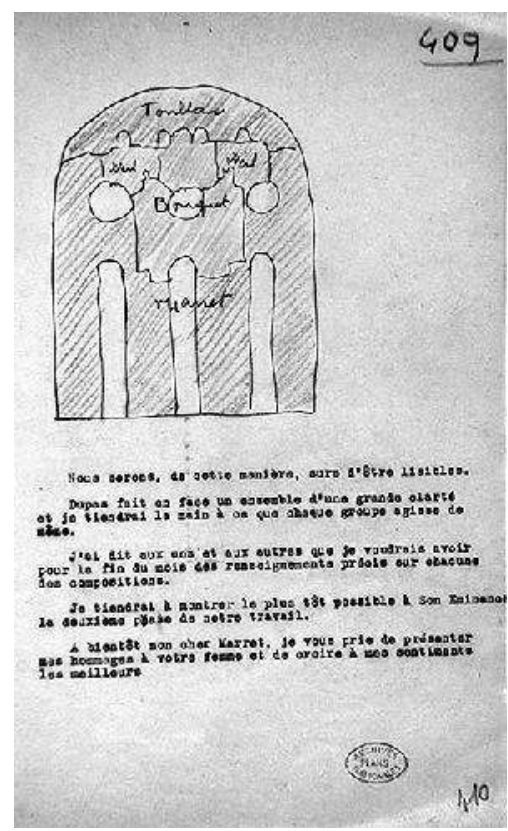

Ill. 16: Paul Tournon, Lettre à Henri Marret, 1932, encre noire et crayons de couleur sur papier dactylographié. Arch. nat., 377-AP/276-409 (photographie : Fouin Christophe (C) Ville de Paris, COARC, 2002. Base mémoire: DSC_2990.JPG).

décor de la dernière chapelle, sous la direction de Valentine Reyre. À Raymond Virac, la troisième; à Marthe Flandrin, pour les Catholiques des Beaux-Arts, la quatrième ; à Jean Dupas, la cinquième, tandis que des élèves de l'École sont répartis entre les différents groupes et Maurice Denis ${ }^{77}$. Tournon convoque et rencontre collectivement les artistes avant l'établissement des esquisses. Il demande au cardinal Verdier de venir à son agence examiner l'ensemble des études au $1 / 10^{\mathrm{e}}$ et les premiers dessins des cartons définitifs, cette réunion devant en outre permettre à chaque artiste de «se rendre compte des voisinages». Une autre visite du cardinal est organisée en mars 1933, Tournon demandant aux artistes de fournir les cartons de leur projet à l'échelle afin de pouvoir les présenter in situ en les piquant aux murs $^{78}$.

Alors que les principaux chantiers religieux menés autour de la Grande guerre sont pour la plupart l'œuvre d'architectes des Monuments historiques, ceux de la période suivante sont confiés par l'archevêché de Paris à des hommes pour qui «la

77. Arch. nat., 377 AP 294.

78. Arch. nat., 377 AP 276, f 122, 23 juillet 1932, lettre circulaire à de Maistre, Flandrin, Marret, Reyre ; Arch. nat., 377 AP 276, f 534, 17 novembre 1932 à Louis Bouquet ; Arch. nat., 377 AP df 538, id. à Marret ; Arch. nat., 377 AP 207, f 223, f 207, f 208, 13 février 1933, lettre circulaire et lettres particulières à Charlier et Toublanc. 
construction d'une église est un acte de foi et non la concrétisation de l'étude ou de la restauration d'églises anciennes " ${ }^{79}$. Ce sont ces "qualités toutes chrétiennes » que reconnaît Yves-Marie Froidevaux (1907-1983) dans l'œuvre de Tournon. L'hommage rendu par Georges-Henri Pingusson (1894-1978) fait de lui un lointain descendant des bâtisseurs de cathédrales, " animant une équipe fervente et passionnée ${ }^{80}$.

Sans être un théoricien de l'art de bâtir, Tournon n'en a pas moins exposé sa conception, spiritualiste, de l'architecture et de l'art religieux. Selon lui, si les antiques voûtes romanes suscitent toujours la même émotion, "c'est qu'elles sont ouvres exclusives de prière, d'offrande, de glorification ". A contrario, rejoignant Jacques Maritain, il qualifie les copies du XIX ${ }^{\mathrm{e}}$ siècle de pastiches ${ }^{81}$. Ceux-ci "intéressent peut-être quelques esprits curieux, ils n'ont jamais fait battre un seul cœur " ${ }^{82}$. Tournon confie en outre s'être "toujours laissé diriger dans [son] étude des édifices religieux par trois choses : le vocable, l'emplacement ou son cadre, et le matériau de construction " ${ }^{83}$. Le cas du Saint-Esprit, où la vision de départ, associée à la dédicace, détermine le programme architectural et décoratif, illustre remarquablement les principes ici énoncés. C'est vraisemblablement par la compréhension de la cathédrale de Marseille que Tournon a faite sienne cette conception rationaliste de l'architecture, Vaudoyer ayant tenu « à ce que son projet soit adapté à l'époque, aux usages locaux, au terrain, bref à cette trilogie des fonctions éminemment labroustiennes. Dès lors, la cathédrale idéale n'est plus un type [...], elle devient dans l'esprit de Vaudoyer une méthode architecturale qui se substitue à une solution ne varietur " ${ }^{84}$. Tournon décline les formes architecturales de ses constructions religieuses autour de deux ou trois des critères ci-dessus évoqués, y ajoutant une référence au vocable, chaque programme engendrant une forme spécifique synthétisant discours architectonique et discours décoratif : clocher sculpté à Villemomble, portail d'inspiration gothique à Sainte-Jeanne d'Arc en raison du contexte historique local, chapelle reliquaire à Élisabethville pour un édifice à vocation votive ; façade et couverture évoquant l'Extrême-Orient, clocher évoquant les minarets d'Afrique noire et bâtiment oblong citant les constructions marocaines au Pavillon des missions, synthèse coloniale; édifice en forme de navire à Toulon ; nef en carène de bateau et clocher-phare à Boulogne-sur-Mer; de même au SaintEsprit, dont la coupole doit évoquer l'Esprit saint ; les clochers-minarets au Maroc, les décrochements successifs centrés symbolisant la tiare papale et la couronne de Marie au Pavillon pontifical, etc. C'est fort de cette adaptation que Paul Tournon puise dans les traditions structurelles et le vocabulaire formel du passé. Outre la

79. Texier dir., p. 54-55.

80. Cat. Paul Tournon, architecte (1881-1976), Paris, éd. Dominique Vincent, 1976, p. 76, ibid., p. 7.

81. Jacques Maritain, Art et Scolastique, Paris, éd. Rouart, 1926, p. 214.

82. Paul Tournon, "Architecture ", L'Art Sacré, n 1, juillet 1935, p. 6-7.

83. P. Tournon, "Architecture religieuse ", Bulletin de la Société des Architectes, no 5, 1938.

84. Jean-Michel Leniaud, dans Chantal Bouchon, Catherine Brisac, Nadine-Josette Chaline, Jean-Michel Leniaud, Ces églises du dix-neuvième siècle, Amiens, Encrage, 1993, p. 72. 


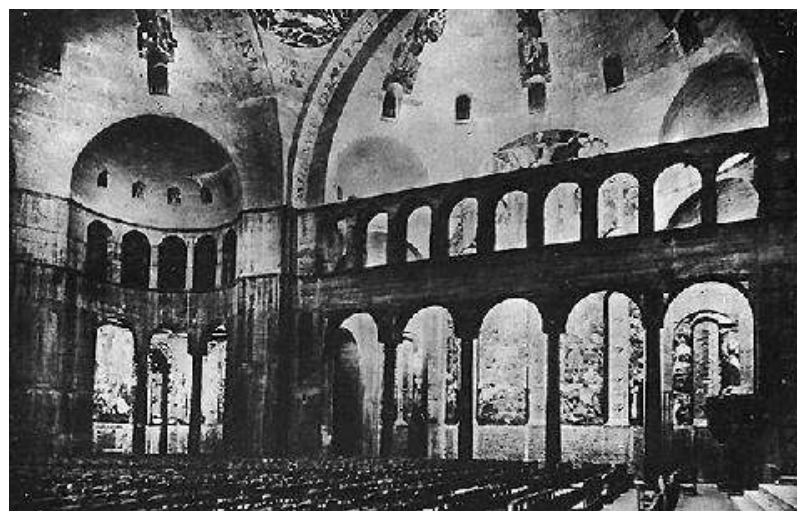

Ill. 17 : Le transept et le triforium de l'église du Saint-Esprit, 1934. Photographe anonyme. Arch. paroissiales (reproduction : Fouin Christophe (C) Ville de Paris, COARC, 2001. Base mémoire : 00_ESP_ 123_972x.JPG).

référence à l'Église chrétienne dans ses premières grandes manifestations officielles - à l'instar de ses contemporaines Sainte-Odile et Saint-Pierre-de-Chaillot -, un souci de simplicité guide les emprunts pour le Saint-Esprit, où il se montre également sensible à la grandeur romaine dans le traitement de l'immense galerie qui enserre la nef ${ }^{85}$ (ill. 17). Cette "romanité " s'y exprime également par l'usage du béton, technique de construction par masse et compression dont dérive partiellement l'architecture byzantine monumentale. L'intérêt de Tournon pour l'art byzantin n'est pas nouveau et il interprète largement l'architecture de Sainte-Sophie ${ }^{86}$. L'étagement des coupoles renouvelle l'effet d'apesanteur, la coupole centrale, basse et enveloppante, ceinturée d'une couronne lumineuse, constituant comme l'expression de l'Esprit saint, le coeur de la composition autour de laquelle Tournon construit un système hiérarchisé, dans lequel chaque élément participe à la statique de l'édifice et au discours symbolique ${ }^{87}$. La silhouette extérieure est proche, bien que l'assise du Saint-Esprit soit plus ramassée et resserrée, les dômes étant comme maintenus en périphérie par les parements verticaux très tendus des rues Cannebière et Claude Decaen.

En Occident, sa préférence va de l'art roman aux débuts de la Renaissance, tant pour le mode d'inscription du décor peint dans l'architecture que dans les formes. La référence à la verticalité austère des parements en brique de Sainte-Cécile d'Albi est patente rue Cannebière, l'escalier d'accès à la cathédrale du Puy a vraisemblablement inspiré celui à l'église haute depuis l'avenue Daumesnil (ill. 18). Cette combinaison "éclectique » est justifiée par la topographie et la contiguïté des deux

85. L'Art sacré, no 1, juillet 1935, "Architecture », par Paul Tournon, p. 6.

86. Arch. nat., 377 AP 207, f 311, 26 mars 1934.

87. Giorgio Pigafetta, Antonella Mastrorilli, Paul Tournon architecte (1881-1964). Le "Moderniste sage", Mardaga, Sprimont, 2004, p. 140 


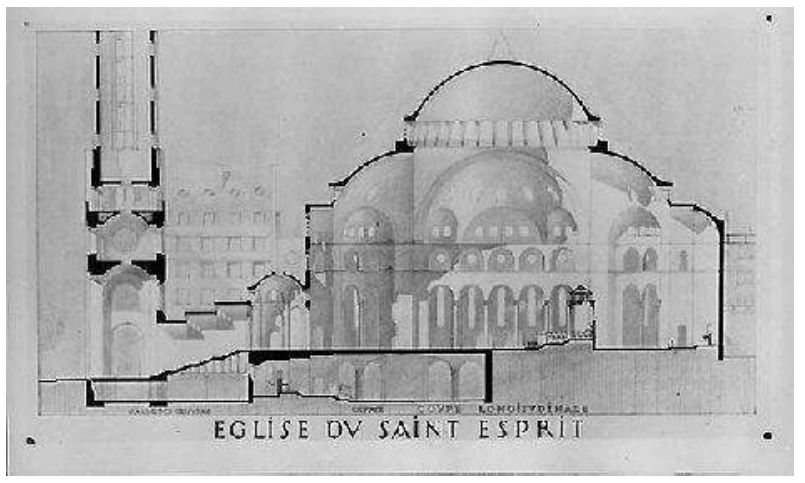

Ill. 18: Paul Tournon, Église du Saint-Esprit, coupe longitudinale, 1929, encre de chine et mine de plomb sur papier. Arch. paroissiales (photographie : Fouin Christophe (c) Ville de Paris, COARC, 2001. Base mémoire : 70_ESP_5_37x.JPG).

parcelles, autorisant et nécessitant tout à la fois cette juxtaposition quasi organique de séquences d'échelle et de nature différentes: l'escalier en boyau, le narthex en rotonde, enfin l'église dilatée. Conformément au mouvement qui se dessine au cours de la décennie, Tournon intègre en outre des innovations liturgiques : à l'instar d'autres édifices contemporains, les fonts baptismaux se trouvaient initialement en contrebas d'une marche dans le sol du baptistère, afin de rappeler la descente dans le Jourdain ${ }^{88}$. Tournon défend un art catholique, fait appel à des artistes catholiques. Il s'en explique longuement à Dupas au moment de la sélection des peintres pour le décor du Saint-Esprit ${ }^{89}$. Il aura la même conviction à l'heure de sélectionner les participants au décor du Pavillon pontifical ${ }^{90}$. Il construit le Pavillon des missions et l'église du Saint-Esprit dans un double contexte de triomphalisme colonial et de reconquête intérieure de la banlieue, auquel il adhère et souhaite participer. Fidèle à la vocation universaliste du catholicisme, il a une conception missionnaire de son rôle d'architecte, élaborant des projets tant pour les Missions étrangères que pour le pourtour de la capitale. Plus concrètement, il construit trois églises au Maroc, celle du Sacré-Coun à Casablanca - dont le maréchal Lyautey voulait faire la cathédrale du Maroc (ill. 19) -, Saint-Joseph de l'Océan à Rabat, et le petit sanctuaire d'Ifrane, station estivale des colons du Maroc. Citons également l'église de Thakkek, au Laos (ill. 20).

88. À Sainte-Marguerite-Marie du Perreux par exemple (André Lecomte architecte, $43^{\text {e }}$ Chantier du cardinal), de même qu’à Limé, dans l'Aisne (Julien Barbier architecte), ou encore, de Tournon, à l'église des Missions catholiques en 1931.

89. Arch. nat., 377 AP 275, f 398, 2 mai 1932.

90. Arch. nat., 377 AP 214, f 607, 29 octobre 1943. 


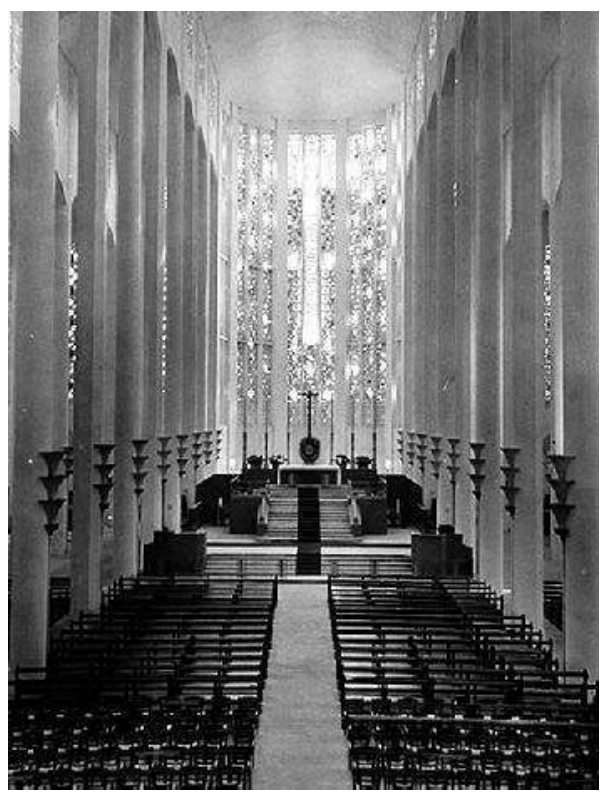

Ill. 19: La cathédrale de Casablanca, vue du choeur, 1930-1946-terminée en 1960. Photographe anonyme. Arch. nat., 377/AP-294 (photographie : Fouin Christophe () Ville de Paris, COARC, 2002. Base mémoire : DSC_8946.JPG).

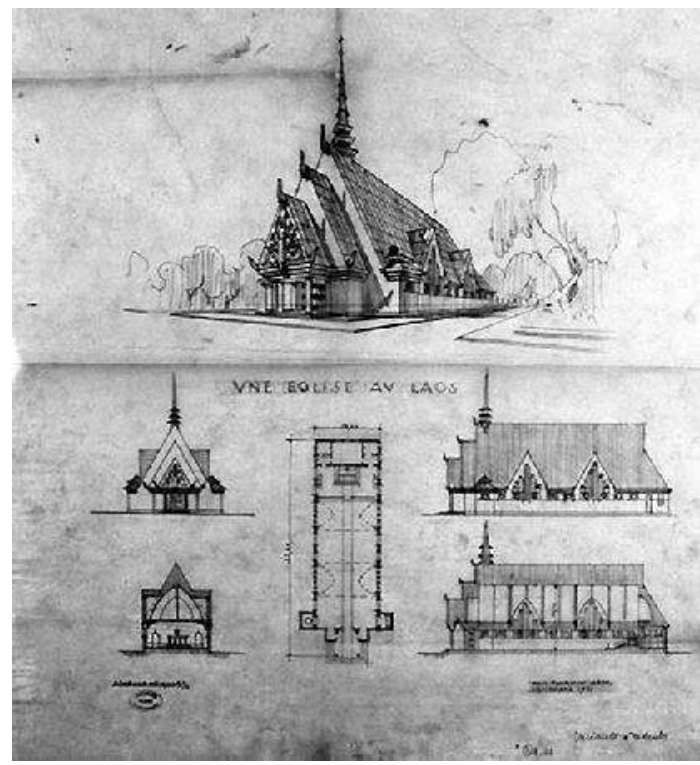

Ill. 20 : Paul Tournon, Projet pour une église au Laos, septembre 1931, mine de plomb sur papier calque, Arch. nat., 377/AP-240 i 2 8-25 (photographie : Fouin Christophe () Ville de Paris, COARC, 2002. Base mémoire : DSC_3405.JPG).

Livraisons d'bistoire de l'architecture $n^{\circ} 27$ 


\section{Tradition et modernité dans l'architecture religieuse}

La problématique de l'alliance de la tradition et de la modernité telle que l'envisage Paul Tournon trouve sa pleine illustration dans l'église du Saint-Esprit ${ }^{91}$. La définition que donne Adorno de la tradition en s'appuyant sur son étymologie latine - tradere, transmettre -, se rapproche de la conception de l'architecte, en ce qu'elle intègre l'idée d'un mouvement tout en excluant la rupture ${ }^{92}$. Pigafetta et Mastrorilli ${ }^{93}$ inscrivent l'ouvre de Tournon dans un courant traditionaliste de l'architecture européenne au XX $\mathrm{XX}^{\mathrm{e}}$ siècle qui fait sa place aux évolutions technologiques, en s'appuyant sur les écrits de Marie Dormoy. Celle-ci, revendiquant le béton armé comme une invention nationale, intègre Perret dans un courant classique usant sans les dissimuler des matériaux et techniques nouveaux, tout en s'enracinant dans la tradition ${ }^{94}$. À l'instar de la démarche définie dès 1980 par Ignasi de Solà-Morales Rubió, nous pourrions élargir la réflexion en déplaçant la compréhension de l'histoire de l'architecture moderne comme avant-garde, vers une conception prenant en considération les processus globaux d'évolution de la sociétép 5 .

La hiérarchisation des projets de l'architecte se combine avec une adéquation formelle et structurelle spécifique à chaque programme important. Alors que le plan centré, à coupoles ou de forme dérivée, semble avoir inspiré Tournon pour les édifices les plus prestigieux - le projet pour la cathédrale de Manizales en Colombie, le Saint-Esprit, la première proposition pour Casablanca, le Pavillon pontifical (ill. 21), puis Saint-Honoré d'Amiens, mais aussi un projet destiné à Saint-Louis de Marseille, en 1933 -, les édifices en banlieue parisienne pour lesquels il disposait de moyens financiers réduits, sont plus simples et de construction sommaire, Tournon leur réservant un plan basilical oblong et une couverture à double pente (ill. 22). Le principe d'une répétition devient alors possible. Il lutte pourtant contre une rationalisation des métiers de la construction menant à une standardisation de l'architecture, et défend le beau métier ${ }^{96}$. Il s'insurge contre les industriels cherchant à investir dans le domaine de l'architecture religieuse en proposant des églises standard, dénonce l'industrie du vitrail à laquelle se livreraient certains maitres-verriers, et se montre opposé au principe de la série dans les œuvres d'art, les voulant originales dans une église ${ }^{97}$. L'idée même du projet-type, développée

91. Albert Louvet, L'Architecture, 15 octobre 1934, p. 355.

92. Parva Aesthetica, Francfort-sur-le-Main, Suhrkamp Verlag, 1967, p. 29, traduction de Giorgio Pigafetta, dans Pigafetta/Mastrorilli, p. 12.

93. Leur démarche s'inscrit dans une historiographie déjà longue. Cf. Ignasi de Solà-Morales Rubió, infra, note 95 .

94. L’Architecture française (1938), Paris, éd. Vincent, Fréal \& $C^{\mathrm{ie}}, 1951$, p. 138.

95. Ignasi de Solà-Morales Rubió, Eclecticismo y vanguardia. El caso de la Arquitectura Moderna en Catalunya, ed. Gustavo Gili, Barcelone, 1980, coll. Arquitectura y Critica, Introducción, p. 9-20.

96. Arch. nat., 377 AP 275, fol. 270, 21 mars 1932, lettre au cercle des Maçons et des tailleurs de pierre.

97. Arch. nat., 377 AP 205, f 411, 27 novembre 1945 à Laforgue, Rabat, à propos des frères Mauméjean ; Arch. nat., 377 AP 275, f 272, 21 mars 1932, rapport au P. Touzé. 


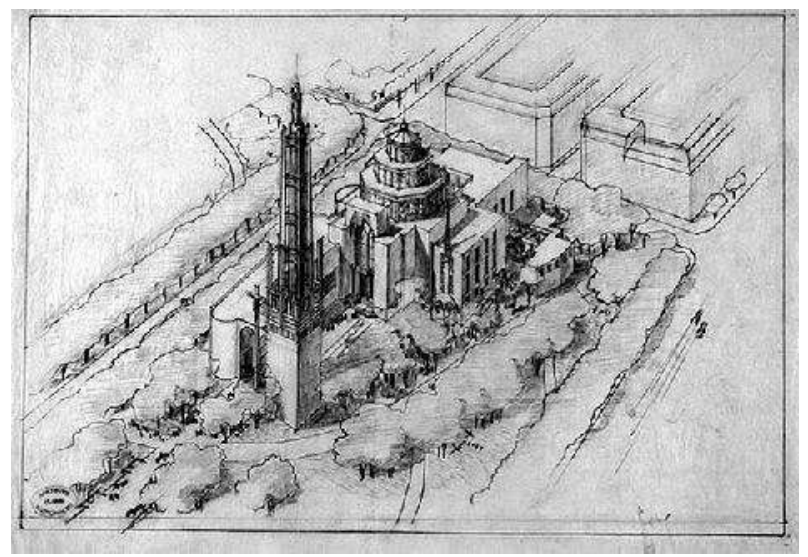

Ill. 21 : Paul Tournon, Le pavillon pontifical à l'Exposition internationale de 1937, vue cavalière, 1937, mine de plomb sur papier calque. Arch. nat., 377/AP- 266 y $13-39$ (photographie: Fouin Christophe (c) Ville de Paris, COARC, 2002. Base mémoire: DSC_2978.JPG).

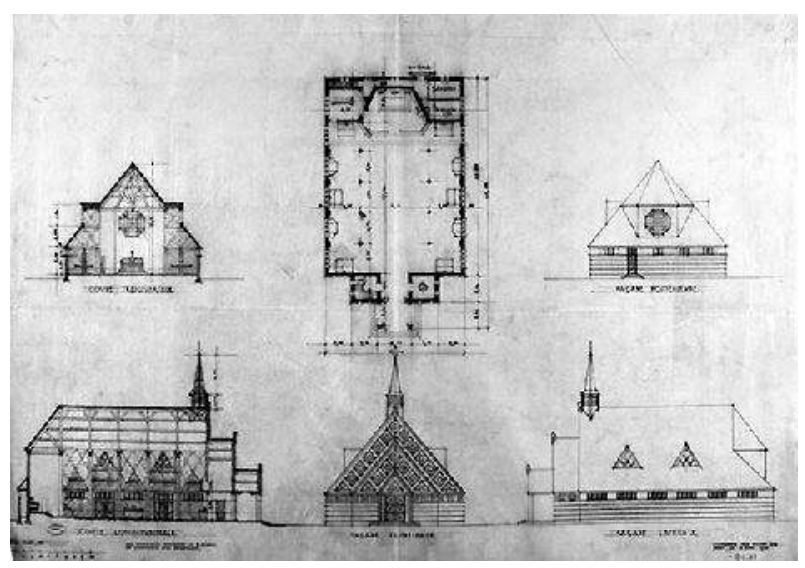

Ill. 22 : Paul Tournon, Projet pour une chapelle de secours en banlieue, 8 juin 1934, tirage en sepia. Arch. nat., 377/AP-240 i $24-13$ (photographie: Fouin Christophe (C) Ville de Paris, COARC, 2002. Base mémoire : DSC_3360.JPG).

au XIXe siècle, lui reste étrangère ${ }^{98}$. Tournon s'inscrit dans une autre logique, celle de la récupération des matériaux. Sous cet angle, il insère une partie de son activité dans une économie traditionnelle du remploi. Ainsi accepte-t-il d'étudier la proposition d'un curé de Cambrai, consistant à installer au Pavillon pontifical des verrières destinées à son église et qui seraient exécutées par Marguerite Huré (1895-

98. Jean-Michel Leniaud, "Les constructions d'églises sous le second Empire : architecture et prix de revient ", dans Revue d'Histoire de l'Église de France, 1979, p. 267-278. 
1967), projet resté sans suite et relayé par celui de la création de vitraux pour la cathédrale Notre-Dame ${ }^{99}$.

Tournon apprend à tirer parti des apprentissages liés à la mise en ouvre d'une architecture éphémère avec le Pavillon des missions catholiques à l'Exposition coloniale. Certains éléments du décor de la chapelle sont transférés à Notre-Dame des Missions, à Épinay-sur-Seine, les autres matériaux utilisés étant provisoires - charpentes en bois revêtues de plaques de fibro-ciment, façade édifiée avec le même matériau et décorée d'une peinture cellulosique, le Duco, imitant la porcelaine chinoise -, tandis que la construction à Épinay est en béton armé et maçonnerie. Tournon a l'intelligence de l'éphémère et sait faire d'une exposition internationale un lieu d'expérimentation, un laboratoire d'architecture ${ }^{100}$. La rapidité de l'édification, vecteur privilégié de diffusion, la confrontation avec la production internationale donnent de l'audace à un architecte averti. Tournon procède à un recyclage des plans et des structures constructives : ainsi du Pavillon des missions à Épinaysur-Seine, de Sainte-Thérèse d'Élisabethville à la cathédrale de Casablanca, du Pavillon pontifical à Saint-Honoré d'Amiens. Il tente de pérenniser ses travaux au moyen de constructions moins prestigieuses mais participant au programme diocésain de construction de lieux de culte dans la banlieue : la vente sur place des matériaux non utilisés du Pavillon des missions, le démontage et le recyclage de constructions provisoires de l'Exposition en de modestes chapelles de secours s'inscrivent dans une économie de la récupération.

La question du prix de revient, si importante à l'heure des Reconstructions ou des Chantiers du cardinal, est ponctuellement abordée. S'agissant de l'emploi du béton, les raisons économiques sont d'emblée énoncées comme déterminantes, avec la construction du clocher de Villemomble. "La sculpture exécutée pour environ 70000 francs, eût coûté, dans la pierre ou le granit, plusieurs centaines de mille francs avec les modèles, les moulages, les mises au point, les praticiens, etc. " ${ }^{101}$. De plus, l'économie de temps est considérable : trois mois au lieu de plusieurs années.

Bien que seules trois des chapelles de secours aient finalement été construites, l'idée initiale était de perpétuer le Pavillon des missions sous la forme de petits édifices autour de Paris selon cinq projets rappelant chacun l'architecture des cinq continents $^{102}$, la récupération de certains éléments permettant de réduire de moitié le prix de revient de ces chapelles. Concernant la construction de Saint-Pierre à Alfortville, le coût de construction devait s'élever, honoraires compris, à 300000 francs environ, ce qui représente avec dépendances et clocher, une moyenne infé-

99. Arch. nat., 377 AP 215, f 497, 18 mai 1937, à l'abbé Crombez; Caroline Piel, « La querelle des vitraux ", Monumental, 2000, dossier Notre-Dame de Paris, p. 54-61; Arch. nat., 377 AP 215; Arch. nat., 377 AP 214, f 607, 29 octobre 1943.

100. Entretien avec Marion Tournon-Branly, 12 décembre 2003.

101. Albert Louvet, "Le clocher de l'église de Villemomble ", L'Architecture, t. XL, nº 8, 1927, p. 233240.

102. Arch. nat., 377 AP 199, f 538, $1^{\text {er }}$ décembre 1931, à l’Amiral Lacaze, Arch. nat., 377 AP, f 286, 6 août 1931 à Fabre ; Arch. nat., 377 AP 275, f 532, 28 novembre 1931 à l'entrepreneur Gillet. 
rieure à 1000 francs le $\mathrm{m}^{2}$, soit «moins que le prix d'un étage courant de maison de rapport" pour une église entièrement voûtée ${ }^{103}$. Le même type de comparaison donne en 1933 un prix de revient de 2500 francs le $\mathrm{m}^{2}$ pour le Saint-Esprit, soit une somme "légèrement inférieure au prix au mètre carré de deux étages d'un bon immeuble de rapport à Paris " ${ }^{104}$. La proposition de Tournon trouve un écho dans la politique de l'archevêché. Après 1931, celui-ci tente de rationaliser sa politique de construction, tant par la concentration des moyens financiers, que par le biais d'une vaste communication autour de la création de l'œuvre des Chantiers du cardinal. Tournon adhère à l'entreprise en faisant valoir le caractère exaltant d'un transfert de l'esprit missionnaire à la banlieue de Paris. Un malentendu s'installera toutefois entre l'architecte et l'abbé Touzé, vicaire général et directeur des Chantiers, qui cherche à construire le maximum d'édifices au moindre coût ${ }^{105}$.

Outre l'argument du prix de revient, l'architecte se livre à un plaidoyer en faveur du béton pour des raisons d'opportunité pratique mais aussi d'ordre esthétique: grâce aux progrès effectués dans sa mise en œuvre, l'usage de ce matériau se généralise dans le bâtiment dans l'Entre-deux-guerres, au point que l'histoire du béton armé et celle de l'architecture moderne vont finir par se confondre. À la modernité canonique des années 50, alors plaisamment qualifiée de nudiste, l'historiographie plus récente a, grâce à une réévaluation plus ouverte, procédé à l'intégration de celle en vogue dans les milieux des Chantiers du cardinal, précisément autour de la figure de Paul Tournon ${ }^{106}$.

Au Saint-Esprit, outre le fait que Tournon est fier de la performance de la grande coupole, l'absence de traitement après le décoffrage de celle-ci participe de l'ambiance décorative. La plupart des matériaux de décor sont employés d'une manière originale, liée principalement à l'usage du béton, qu'il s'agisse d'une réappropriation de techniques traditionnelles, ainsi de la mosaïque noyée dans le béton; voire du réapprentissage d'une technique oubliée, avec la fresque ${ }^{107}$. Il en est de même du recours au verre inséré en double feuillure dans les claustras de ciment selon plusieurs modules ${ }^{108}$. La brique est utilisée comme matériau de recouvrement du béton sur un mode décoratif, jouant sur le dessin du calepinage et des modénatures ${ }^{109}$. Il est fait recours au Stic B comme isolant, mais surtout

103. Arch. nat., 377 AP 207, f 550, 11 juin 1934 à L'Architecture d'aujourd'hui ; Arch. nat., 377 AP 207, 5 octobre 1933, à Norbert Noë ; L'Architecture, mai 1938.

104. Léon de Lapérouse, "L’Architecture catholique moderne ", Le Miroir du monde, 11 novembre 1933 , p. 483.

105. Cf. les projets-types présentés dans la revue des Chantiers, Christ dans la banlieue (1927-1931).

106. Simon Texier dir., op. cit.

107. Arch. nat., 377 AP 207, f 508, $1^{\text {er }}$ mai 1933, à Charlier.

108. Élisabethville, Saint-Esprit, Ifrane. Marguerite Huré, qui avait travaillé avec Maurice Denis au Prieuré en 1922, puis collaboré avec Perret et le peintre au Raincy en 1925, travaille pour Tournon à Élisabethville en 1928 puis, au Pavillon des missions en 1931. Elle retravaillera avec Perret au Havre dans les années 1950 (information fournie par Véronique David).

109. De nombreux architectes s'emparent alors de cette tradition constructive du nord de l'Europe et de l'Espagne, à l'instar de Dom Bellot, Maurice Storez ou Duval et Gonse. 
comme medium $^{110}$; de même pour le Silimat de Van Malderen ${ }^{111}$. L'utilisation de l'aluminium martelé pour les bénitiers de Richard Desvallières constitue une première, tout comme le revêtement de la charpente de Notre-Dame des Missions à l'aide d'un matériau s'apparentant au celluloïd recouvert d'une mince feuille d'argent guilloché. Sur la façade du Pavillon des missions, le Duco est appliqué sur des plaques en fibro-ciment. La sculpture est réalisée en béton moulé ou de préférence taillée dans le béton frais par Sarrabezolles. Grâce au moulage, cette mise en œuvre autorise également des hardiesses dans l'élancement des figures, la dentelle des claustra, recherches d'une osmose entre décor et expressivité de la structure.

L'architecte exalte la possibilité de formes nouvelles en exploitant au maximum la nature plastique et stéréométrique du béton, qui se coule et se moule aisément, associant les qualités de matériaux résistant à l'écrasement, à celles de ceux travaillant à la flexion et à l'extension ${ }^{112}$. Ainsi à Élisabethville, les parois ne jouant aucun rôle portant, l'ossature en béton armé autorise le déploiement d'un jeu de claustras laissant une large place au vitrail ${ }^{113}$ (ill. 4). Cette relecture de la SainteChapelle de Paris nous amène à examiner de plus près les relations entre Tournon et Perret.

Marion Tournon-Branly rapporte qu'en début de carrière, à l'occasion de leur participation au concours de Sainte-Jeanne d'Arc, Tournon « eut le coup de foudre pour le projet de Perret ». Bien que les relations d'amitié véritable se soient nouées ultérieurement par l'intermédiaire de la jeune fille qui fut l'une des élèves de Perret à partir de 1942, une estime réciproque prévalut toujours entre eux ${ }^{114}$. Tournon

110. Cf. supra; marque déposée en 1916 par Pierre Bertin Goyvaerts, commercialisée ensuite par lui et Alice Lapeyre. Cette peinture, appréciée pendant plus de trente ans par de nombreux artistes peintres et architectes pour son rendu (aspect mat de la fresque, vivacité des coloris) et ses qualités de conservation, applicable directement sur des bétons et enduits de ciment, ainsi que sur le métal, est à base de pigments en suspension dans un medium comprenant de l'huile de lin cuite, une gomme dure à vernis, des oxydes de zinc et de titane (Jean Rudel, Techniques de la peinture, PUF, 1983). La marque a été rachetée par Lafarge-Peintures, mais les archives furent détruites entre 1996 et 1998. Maurice Denis avait déjà employé ce medium à Saint-Louis de Vincennes, en 1927, pour La Glorification de saint Louis peinte à l'abside.

111. Matériau siliceux destiné à favoriser le durcissement et la préservation de l'enduit, déposé et commercialisé par un fabricant de chaux, ciments, briques et tuiles. Mentionné dans "L'Église du Saint-Esprit ", Les Guides de l'art sacré, s.d. ; une autre publication (Paul Tournon, "L'Église du Saint-Esprit ", L'Architecture, n 7 , janvier 1936, p. 17) fait en revanche état de l'emploi de caséine, matière organique qui n'est pas conforme aux analyses physiques effectuées en 1999 lors de la restauration du baptistère : Catherine Leenhardt, Françoise Vernochet, Église du Saint-Esprit, restauration des peintures du baptistère, 1999-2000, Conservation des œuvres d'art religieuses et civiles.

112. L'Art sacré, no 1, juillet 1935. Arch. nat., 377 AP 228, f 188, 12 décembre 1928, "Le béton armé », 3 p. dactyl. : "Organisme vivant possédant des os et des muscles [...] dans une enveloppe de chair [...], il a toutes les souplesses de la vie. »

113. Gwenaël Delhumeau, "La nouvelle jeunesse de Sainte-Thérèse d'Élisabethville ", La Construction moderne, $\mathrm{n}^{\circ} 40$, mars 1987 , p. 7838.

114. Entretien du 12 décembre 2003 ; sur cette amitié, outre ce témoignage, cf. aussi Paul Tournon, architecte, 1881-1964, Institut de France, Académie des Beaux-Arts, 1969, catalogue d'exposition, Musée de Caen, texte d'Yves Brayer ; Tournon, "Perret ", La Construction moderne, no 2, 1962. 
connaissait bien les travaux de Perret, y compris dans leurs aspects problématiques, et défendit toujours celui-ci dans les instances professionnelles. Ainsi, à la suite de l'ouverture par son confrère de l'Atelier du palais de bois ${ }^{115}$, Tournon fut le seul à voter pour les projets présentés aux concours par les étudiants de Perret. Les deux hommes se fréquentèrent principalement à partir du second conflit mondial, et Tournon s'engagea en 1943 en faveur de la candidature de Perret à l'Académie ${ }^{116}$. C'est que Tournon se situe lui-même au sein de l'école moderne classique, celle des « œuvres de premier plan ayant marqué [...] l'évolution de l'architecture, comme la galerie des machines, les hangars d'Orly, le théâtre de Perret " ${ }^{117}$. En un sens, les deux hommes ont suivi des cheminements parallèles, dans une vision structurelle de l'architecture mais, alors que Tournon sentait les structures, Perret a inventé un système ${ }^{118}$. Une commune source d'inspiration méditerranéenne les rapproche sans doute $^{119}$ : en 1954 , il évoque " ce sentiment d'éclairement intérieur soudain qu'un mot de lui pouvait produire. C'est qu'il s'y trouvait à la fois la raison sereine des sages de l'Attique et l'audace tranquille des grands bâtisseurs de chez nous ", la Sainte-Chapelle de Paris constituant l'autre référence commune ${ }^{120}$. La chapelle Sainte-Thérèse d'Élisabethville présente des analogies formelles avec l'œuvre du Raincy, que Tournon admirait. Toutes deux sont des cages de verre. Gérard Benoît livre peut-être la clé de la fascination de Tournon pour le «verbe droit, clair, laconique et sans compromission" de Perret, et une "conception jamais dissociée de l'exécution ", l'architecte se doublant d'un entrepreneur, dont l'apport principal au milieu d'un enseignement encore totalement voué à l'image consiste à avoir été si profondément "constructeur " ${ }^{121}$. Mais alors qu'au Raincy était expérimentée une synthèse gothico-classique issue du rationalisme de Viollet-le-Duc, à Élisabethville, le baroquisme décoratif de la façade, tout comme celui du clocher de Villemomble, s'appuie sur la pesanteur du matériau. En revisitant le modèle de la Sainte-Chapelle de Paris, Perret engage la transformation de l'espace sacré, tandis que Tournon suit cette référence pour s'orienter vers une conception sculpturale quasi expressionniste de l'architecture. Patente dans l'œuvre religieux d'un architecte qui, enfant, se

115. Atelier ouvert dans le Palais de Bois - construit en 1924 pour abriter les expositions du salon des Tuileries -, à la demande d'étudiants en architecture lassés par l'enseignement académique. Cf. Architectes, n 52, novembre 1974, cahier spécial Perret (1875-1954), p. 11-13, André Hermant, "Le musée ne doit pas être un labyrinthe".

116. Arch. nat., 377 AP 214, f 91, 7 février 1943, à Tournaire, membre de l'Institut, en réaction à une lettre de Jacques-Émile Blanche publiée dans la presse.

117. Arch. nat., 377 AP 215, f 880, lettre du 22 novembre 1937 à son confrère Bluysen, président de la Société des architectes modernes, au sujet d'un courrier de Louis Hautecœur prévoyant, dans le cadre d'une exposition d'architecture dans les nouveaux musées, la présentation d'œuvres d'architecture moderne: Tournon propose, si un dispositif pérenne est envisagé, de présenter les œuvres ci-dessus mentionnées, dont il invoque le caractère classique.

118. Marion Tournon-Branly, op. cit.

119. La Construction moderne, 1962, no 2 (78 e année), p. 23, Paul Tournon, "En l'honneur d'Auguste Perret ", discours pour l'inauguration d'un buste de Bourdelle à l'effigie d'Auguste Perret.

120. Paul Tournon, "L'audace tranquille d'un bâtisseur", Architectes, op. cit., p. 27.

121. Architectes, op. cit., "Concordances", p. 13. 


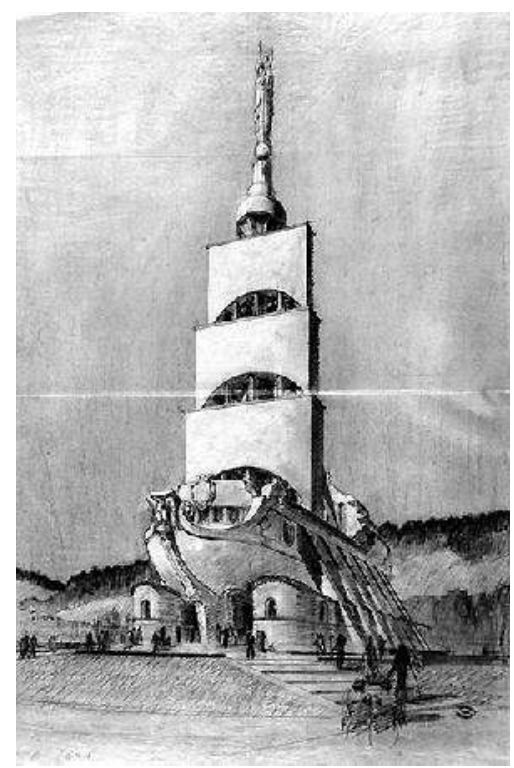

Ill. 23 : Paul Tournon et Henri Chapon, Projet pour Notre-Dame des marins à Toulon, 1942-1943, mine de plomb et gouache blanche sur papier calque. Arch. nat., 377/AP-232 y 1 13-03 (photographie: Fouin Christophe @ Ville de Paris, COARC, 2002. Base mémoire : DSC_2971.JPG).

rêvait sculpteur, cette "passion pour la plastique " 122 amène Tournon à repenser les rapports entre architecture et sculpture, tentation à rapprocher de celle de son âné Gaudí, ce fils de chaudronnier qui conçut ses parements de pierre comme des parois douées de plasticité et voua les trente dernières années de sa vie à l'élévation du plus grand édifice sacré de Barcelone, support géant d'une sculpture surabondante et débridée. Paul Tournon allait proposer, quant à lui, un exemple limite d'architecture avec son projet pour Notre-Dame des Flots au Mont-Faron, en $1942^{123}$ (ill. 23).

À la différence d'Auguste Perret cependant, il revisite l'architecture des grandes églises byzantines dans une attitude qui ressemble à celle des éclectiques du XIX ${ }^{e}$ siècle qui surent, à l'instar de Baltard à Saint-Augustin, utiliser le fer, matériau nouveau, inventant une forme nouvelle d'église déterminée par la forme de la parcelle, c'est-à-dire par des contraintes extérieures, tout en ayant recours à un répertoire formel ancien et multiple ${ }^{124}$. Ce jugement mérite d'être nuancé, la foi

122. Philippe Ollier, Paul Tournon - Architecture religieuse (1881-1964), mémoire de DEA d'histoire de l'art, Bruno Foucart dir., Université de Paris-IV-Sorbonne, 2004, p. 56.

123. Philippe Ollier, op. cit., p. 58. Bien que Tournon soit allé à plusieurs reprises à Barcelone, notamment pour visiter l'Exposition internationale de 1929, il ne fait jamais référence à Gaudí, ce qui ne laisse pas de surprendre.

124. François Loyer, Histoire de l'Architecture française, vol. 3 : «De la Révolution à nos jours ", Mengès, éd. du patrimoine, Paris, 1999, p. 252-253. 
de Tournon voulant conférer une intensité spirituelle supplémentaire à ses édifices religieux, alors que l'agnosticisme de Perret n'empêche en rien son œuvre d'être empreinte de spiritualité ${ }^{125}$. Il n'est pas anodin que Tournon ait choisi pour modèles la Sainte-Chapelle de Paris, symbole de la foi de saint Louis devenu dès lors un prototype, et Sainte-Sophie de Constantinople, également destinée à devenir un prototype quoique repris par l'Islam en terre ottomane. À chaque fois, le modèle est renouvelé en fonction de la demande du commanditaire. Le clergé ayant demandé que soient disposés les futurs vitraux de Notre-Dame dans le Pavillon pontifical de 1937, qui allait devenir le Pavillon marial après l'Exposition internationale, Tournon s'est basé sur les proportions de ceux-ci. Destinés à glorifier Marie, ils furent conçus comme une couronne de lumière. À la différence des projets et réalisations de Perret - pour le clocher du Raincy, les tours-lanternes de la basilique Sainte-Jeanne d'Arc ou de Saint-Joseph du Havre -, Tournon n'a pas dessiné un signal complet, mais a suivi le thème imposé : le clocher du Pavillon pontifical domine toutefois, symboliquement, les pavillons de l'Allemagne nazie et d'URSS sur la pente de la colline du Trocadéro. C'est le principe de cette tourlanterne tronquée qu'il reprend à Saint-Honoré d'Amiens en 1957. Celui du clocher-phare est en revanche clairement affirmé au Saint-Esprit, a fortiori à Boulogne-sur-Mer. À la transparence légère, presque gracile de Notre-Dame du Raincy s'opposent l'effet de masse, la lourdeur voulue du clocher de Villemomble et l'ancrage de l'église du Saint-Esprit. Bien que le système soit entièrement lisible à Notre-Dame-de-la-Consolation du Raincy, c'est néanmoins Sainte-Thérèse d'Élisabethville qui fut qualifiée de "Sainte-Chapelle du ciment armé " dans la presse de vulgarisation ${ }^{126}$. À travers la prise en compte des contraintes liées au lieu, à la dédicace et à la demande du commanditaire, s'affirme clairement un style.

La question de la nudité du béton intérieur dans l'œuvre des deux architectes a fait l'objet d'interprétations abusives, voire anachroniques (cf. supra). Le béton est certes brut au Raincy mais selon Simon Texier, la question d'une éventuelle décoration reste posée ${ }^{127}$. La même ambiguïté est valable au Saint-Esprit ${ }^{128}$. Dans les deux cas, la nudité pourrait bien être partiellement due au manque de moyens. Dans les édifices religieux contemporains, lorsque le béton est laissé apparent il est traité - moulage très soigné, bouchardage, sculpture - afin de lui conférer une apparence plus "noble » ${ }^{129}$ et ce n'est que plus récemment que le brutalisme du matériau a été exalté. Néanmoins Tournon a vite faite sienne l'idée du béton nu, revendiquant une recherche de "la beauté, toute crue, dans le béton armé " ${ }^{130}$.

125. André Le Donné, "Le Sacré dans l'œuvre de Perret ", Architectes, n 52, novembre 1974, cahier spécial Perret (1875-1954), p. 8-10.

126. Lectures pour Tous, septembre 1928.

127. Simon Texier dir., op. cit., p. 78 .

128. "Conférence de M. Tournon ", Le Béton armé, janvier 1931, p. 607.

129. Exemples : à Saint-Christophe de Javel, le béton est très soigneusement moulé, dans des moules finement travaillés. À Saint-Pierre de Chaillot, il est entièrement bouchardé.

130. Arch. nat., 377 AP portef. 231/2, f n $12-225$; anonyme, Église du Saint-Esprit, Mémorial permanent de l'Action missionnaire, s.d., p. 6. 
De Villemomble au Saint-Esprit, c'est au cours des années 1925 à 1931, période la plus innovante dans le domaine de l'architecture religieuse, que Tournon met en place toutes les formules architecturales développées ultérieurement, inaugurant des solutions tant techniques, que formelles et décoratives. Sa capacité d'innovation peut alors se déployer dans des programmes où la technique et l'obligation de fonctionnalité sont primordiales : laboratoire d'Édouard Branly, sanatoria en collaboration avec Louis Feine (1868-1949), où le fonctionnalisme est décliné en métaphores hygiénistes et aériennes. Cette capacité d'affranchissement vis-à-vis du passé est à mettre en parallèle avec le rationalisme d'un Perret, d'un Tony Garnier. Dans le même temps, la modération dans le rapport à la modernité observable dans l'architecture religieuse inscrit son œuvre dans l'«école française ", à l'instar de la Reconstruction, au travers notamment d'une identification régionale, associée à l'usage du béton armé, tandis qu'un goût prononcé pour la sculpture fait de lui le parent d'une certaine forme d'expressionnisme.

L'œuvre de Paul Tournon confirme l'hypothèse selon laquelle le béton serait le matériau de l'éclectisme dans le premier $\mathrm{XX}^{\mathrm{e}}$ siècle, tant dans une interrogation stylistique que dans une démarche constructive. Tournon affirme sa maîtrise au sein de l'affrontement entre entrepreneurs, ingénieurs et architectes qui tentent, au nom de leurs compétences respectives, de prendre l'ascendant sur le développement de cette technologie. Dans un autre ordre d'idées, sa conception de la restauration, respectueuse de l'existant de diverses époques, s'inscrit dans le sillage de Lassus. Cette cohérence appuie une synthèse des arts qu'autorise une conception tout à la fois monumentale et intime du rapport entre le décor et l'architecture. Aux colonies, dans un contexte de triomphalisme empreint de paternalisme, Tournon fait preuve d'un certain intérêt pour les formes et parfois les matériaux traditionnels, et d'une relative prise en compte des contraintes climatiques. A contrario, le cas du Pavillon des missions catholiques à l'Exposition coloniale de 1931, totalement hors contexte, constitue un renversement paradoxalement "pittoresque ». L'expérience coloniale comme lieu de mise en pratique, est à mettre en perspective avec une forte implication dans la dimension missionnaire de la pastorale de l'Église catholique dans la banlieue de Paris.

L'église du Saint-Esprit occupe ainsi une place centrale dans l'œuvre de l'un des principaux protagonistes du débat sur l'église moderne et l'espace sacré, et constitue la principale vitrine des Chantiers du cardinal avant guerre. Certes, des églises de taille équivalente furent également construites dans le diocèse de Paris, chantiers qui bénéficièrent d'un financement privé conséquent. Pour autant, avec de moindres moyens, la présence de cet édifice s'impose, en particulier par l'importance du décor peint, malgré - la sensibilité actuelle dirait "en raison de » - son inachèvement.

Paul Tournon délivre en 1943 ses recommandations à un jeune homme souhaitant entrer dans la carrière d'architecte : "développer de tout son pouvoir sa culture générale ", "dessiner beaucoup surtout d'après l'antique qui est la plus haute éducation de l'œil ; et entre ses travaux d'étude [...] regarder les belles choses 
construites non en touriste mais en maître maçon, à la manière de ceux des cathédrales qui apportaient la même ferveur à la conception de leurs œuvres qu'à la manière de les réaliser». Ces conseils alliant discipline des Beaux-Arts et observation pratique résument toute son approche de l'architecture ${ }^{131}$.

Cette étude a voulu mettre en évidence l'enthousiasme expérimental d'un pragmatique mystique.

Miriam SIMON conservatrice en chef responsable du cabinet des arts graphiques Musée Carnavalet - Histoire de Paris

131. Arch. nat., 377 AP 214, d 418, 15 juillet 1943.

Livraisons d'bistoire de l'architecture $n^{\circ} 27$ 


\section{Annexe I - Repères dans l'ensemble de l'œuvre construit de Paul Tournon}

\section{Commandes civiles}

Monuments aux morts (1920-1922), villas, banques, maisons de la radio, châteaux, constructions hospitalières, université (en collaboration), groupes scolaires, maisons d'étudiants, usines, raffinerie de pétrole, pavillons de cités ouvrières, infrastructures de club de golf, laboratoire de son beau-père Édouard Branly à l'Institut catholique (1931-1933), nouveau Palais des expositions, Porte de Versailles (1934). À Élisabethville, outre l'église, plusieurs villas, un restaurant, la piscine et un groupe scolaire.

\section{Architecture éphémère}

Palais des expositions agricoles (1920).

Pavillon de Provence, club des architectes de la SADG (Exposition des arts décoratifs, 1925).

Pavillon des missions catholiques (Exposition coloniale, 1931).

Pavillon de Provence, Palais de l'Union corporative de l'art français, Pavillon pontifical (Exposition internationale, 1937).

Chargé par la Société de Saint-Jean de l'ordonnance architecturale des salles françaises aux expositions d'art sacré à Rome, 1932, 1934, 1942, 1947.

Pavillon de la Société des nations et de la Cour internationale de justice de La Haye (Exposition internationale de New York, 1939).

\section{Restaurations et reconstructions}

Restaurations, aménagement et agrandissement de monuments anciens : chargé au titre des Bâtiments civils, notamment, des archives nationales, et de l'abbaye de Sully à SaintBenoît-sur-Loire. Abbaye de Saint-Wandrille à partir de 1945.

Reconstruction: après la Seconde Guerre mondiale participe, à la tête d'un groupe d'architectes de ses anciens élèves, à la reconstruction d'Amiens, d'Abbeville et d'une partie de la baie de la Somme (secteur où il est également membre de la commission de classement des architectes de la Reconstruction).

\section{Annexe II - Corpus des édifices religieux neufs (constructions et projets)}

Paul Tournon n'édifie pas moins de quatorze chapelles et églises, ainsi que trois autels et deux clochers, sans compter les constructions confiées par l'archevêché de Paris dans le cadre d'expositions et sa participation au concours pour l'église votive de Jeanne d'Arc à Paris. Il travaille en outre à l'extension de couvents bénédictins (Saint-Benoit-sur-Loire, Indre-et-Loire, Saint-Wandrille, Seine-Maritime).

\section{Paris et la région parisienne}

Autel de la chapelle Saint-François d'Assise, crypte du Sacré-Cœur, Paris (XVIII ${ }^{\mathrm{e}}$ arrondissement) [1924 ?].

Maître-autel de l'église Saint-François-de-Sales, Paris, 15-17 rue Ampère (XVII ${ }^{\mathrm{e}}$ arrondissement) [entre 1919 et 1925].

Clocher de l'église de Villemomble (Seine-Saint-Denis), 1926 (projet : août 1925).

Concours pour l'église Sainte-Jeanne d'Arc, Paris (XVIII ${ }^{e}$ arrondissement), 1926 ; $3^{\text {e }}$ prix. 
Chapelle Sainte-Thérèse de l'Enfant-Jésus, Élisabethville (Yvelines), 1928.

Église du Saint-Esprit, Paris (XII ${ }^{\mathrm{e}}$ arrondissement), 1928-1934-1961.

Autel de la chapelle Saint-Thomas d'Aquin, chapelle dominicaine de l'Assomption, 222 rue du Faubourg-Saint-Honoré, Paris (VIII ${ }^{\mathrm{e}}$ arrondissement), 1930 (commande du R. P. Janvier ; statue de Sarrabezolles datée de 1924).

Pavillon des missions, Exposition coloniale, Paris, 1931.

Église Saint-Pierre Apôtre, Ile-Saint-Pierre, Alfortville (Val-de-Marne), 1931-1935.

Projet de chapelle de secours pour Orly (Val-de-Marne), 1932 (non réalisé).

Église Sainte-Jeanne de Chantal, La Garenne, Antony (Hauts-de-Seine), 1932.

Projet pour la chapelle Sainte-Cécile de la Briche, Épinay-sur-Seine (Seine-Saint-Denis), 1932 (non réalisé).

Projet pour l'église Notre-Dame du Calvaire, Châtillon (Haut-de-Seine), 1933 (projet et construction finalement confiés à Laffillée).

Église Notre-Dame des Missions, Le Cygne-d'Enghien, Épinay-sur-Seine (Seine-SaintDenis), 1933-1934.

Pavillon pontifical, Exposition internationale, Paris, 1937. Transformation en Pavillon marial, 1938 (démolition en décembre 1938).

Projet de Carillon du centenaire, dédié à Notre-Dame de France, Suresnes (Hauts-deSeine), 1938-1939 (non réalisé).

Chapelle Notre-Dame de l'Étoile, Bobigny (Seine-Saint-Denis), bénédiction en 1938 (détruite par un incendie en 1978).

Projet de musée de la civilisation chrétienne, Paris, 1939-1940 (non réalisé).

Projet de réaménagement de la chapelle Saint-Symphorien, Saint-Germain-des-Prés, Paris (VI ${ }^{\mathrm{e}}$ arrondissement), 1941 (non réalisé). lisé).

Chapelle de l'Institut Sainte-Marie, Neuilly-sur-Seine (Hauts-de-Seine), 1941 (non réa-

Province

Chapelle d'hospice, Compiègne (Oise), 1919.

Projet de chapelle de pèlerinage pour les automobilistes, Saint-Christophe-le-Jajolet, Mortrée (Orne), 1926-1927 (non réalisé). lisé).

Projet pour l'église Notre-Dame de Lourdes, Romans-sur-Isère (Drôme), 1931 (non réa-

Projet pour Notre-Dame de Trévois, Troyes (Aube), 1930-1931 (projet et construction finalement confiés à Dom Bellot, 1932).

Projet pour Saint-Louis, Marseille (Bouches-du-Rhône), 1933 (non réalisé).

Chapelle du sanatorium de Magnanville, Mantes-la-Jolie (Yvelines), 1932.

1962.

Chapelle du couvent Sainte-Marie de la Visitation, Annecy (Haute-Savoie), 1933-1944-

Église de Mont-Saint-Hervon (Loire-Atlantique), 1935.

Clocher de l'église de Saint-Maixent (Île-et-Vilaine), 1935 (non réalisé).

Projet d'église pour Boulogne-sur-Mer (Pas-de-Calais), non daté (non réalisé).

Projet pour l'église Saint-Léger, Cognac (Charente), 1941 (non réalisé).

Projet Notre-Dame des Marins au Mont-Faron, Toulon (Var), 1942-1943 (non réalisé).

Extension de l'abbaye de Fontenelle, Saint-Wandrille, Caudebec-en-Caux (SeineMaritime), 1943-1967, en collaboration (Marion Tournon-Branly et André Le Donné, après la mort de Tournon, pour la reconstruction de la Grange de Canteloup).

Livraisons d'bistoire de l'architecture $n^{\circ} 27$ 
Projet pour le parvis de la cathédrale, Amiens (Somme), 1943-1944 (non réalisé).

Extension de l'abbaye de Sully, Saint-Benoît-sur-Loire (Loiret), projet 1953 ; première tranche achevée en 1958 - jusqu'en 1965, en collaboration pour l'achèvement (Marion Tournon-Branly et Henry Davy).

Église Saint-Honoré, Amiens (Somme), 1951-1961. Aménagement de l'esplanade Branly autour de l'église.

Colonies et protectorats français, reste du monde

Projet de cathédrale du Christ-Roi, Manizales (Colombie), 1927 (non réalisé).

Église du Sacré-Cœur, dite "la Cathédrale ", ou le Christ-Roi, Casablanca (Maroc), 1930-1946-terminé en 1960 (désaffectée).

Sainte-Marie de l'Océan, Rabat (Maroc), construite sous le vocable de Saint-Joseph de l'Océan, 1927-35 (désaffectée).

Église Notre-Dame des Cèdres, Ifrane (Maroc, Haut-Atlas), 1932-1939.

Projets pour de petites églises en Asie, Afrique, Moyen Orient, Océanie, Amérique, 1931 (non réalisés, hormis une variation avec la petite église de Thakkek, Laos, 1931-1932).

Projets-types pour des églises à Madagascar, 1934 (non réalisés).

Église Notre-Dame, Agadir (Maroc), 1934 (non réalisé).

Église du Christ-Roi, Beyrouth (Liban), 1935 (non réalisé).

\section{Annexe III - Sources et bibliographie}

\section{Instruments de recherche}

Sonia Gaubert (dir.), Marcos Carvalho-Canto, Vlada Filhon et Holy Raveloarisoa, Fonds Tournon, Paul (1881-1964). 351 AA, Centre d'archives de l'Institut français d'architecture, 2002, dépôt de l'Académie d'architecture.

M. Maresca et G. West, Inventaire analytique dactylographié (plans, 377 AP 1-70, cartons 377 AP 71-284), Archives nationales, fonds Paul Tournon, 1978, 78 et 20 p.

[Miriam Simon], Inventaire du décor de l'église du Saint-Esprit, Conservation des œuvres d'art religieuses et civiles, Ville de Paris, 2000.

Claudine de Vaulchier, Catalogue des collections, 1750-1900, vol. II : 1890-1970, Académie d'architecture, 1987, p. 388-389.

\section{Écrits de Paul Tournon publiés}

"Église du Saint-Esprit à Paris", Le Béton armé, no 275, janvier 1931, p. 601-607. Repris dans "L'architecture des églises et l'Église du Saint-Esprit ", Le Message du SaintEsprit, $\mathrm{n}^{\circ} 10$, juillet-août, p. 15-17 et no 11, p. 11-13, 1938.

"Architecture ", L'Art Sacré, no 1, juillet 1935, p. 6-7.

"L'église du Saint-Esprit », L'Art sacré, no 7, janvier 1936, p. 13-18.

L'Église du Saint-Esprit à Paris, Strasbourg, E.D.A.R.I., 1936.

Artisans d'art et de foi à l'exposition de 1937, Paris, 1937, brochure.

"Architecture religieuse », Bulletin de la Société des architectes, nº 5, 1938.

L'enseignement à l'École des Beaux-Arts, séance annuelle des cinq Académies du 26 octobre 1942, Institut de France, Académie des Beaux-Arts. 
Notice sur la vie et les æeuvres de Gustave Umbdenstock (1866-1940), séance du $1^{\text {er }}$ mai 1943, Institut de France, Académie des Beaux-Arts, Paris, 1943.

"L'interdépendance des trois arts dans l'œuvre d'architecture ", Bulletin de la Classe des Beaux-Arts de l'Académie royale de Belgique, t. 18, 1961, p. 126-139.

\section{Bibliographie}

Marcel Aubert, Les Chantiers du Cardinal, Paris, éd. François, 1943.

Urbain Cassan, "Notice sur la vie et les travaux de Paul Tournon (1881-1964) ", Bulletin de l'Académie des Beaux-Arts, no 11, Institut de France, Académie des Beaux-Arts, 1966, p. $44-48$.

Collectif, L'Art sacré en France au XXe siècle, Thonon-les-Bains, Éditions de l'Albaron / Musée et centre culturel de Boulogne-Billancourt, 1993, p. 145-147.

Marie Dormoy, L'Architecture française, [1938], Boulogne-Billancourt, L'Architecture d'aujourd'hui, réédition Édition Vincent, Fréal \& Cie, Paris, 1951.

Maximilien Gauthier, "Les Fresques de l'église du Saint-Esprit ", Art et décoration, t. LXIII, 1934, p. 363-371.

Guide du Pavillon pontifical, L'Art sacré, Paris, 1937.

Charles Imbert, "L'église du Saint-Esprit à Paris", La Technique des Travaux, nº 3, mars 1933 , p. 130-144.

L'Église du Saint-Esprit, Les Guides de l'art sacré, s.d., Paris.

Albert Louvet, "Les églises modernes. Le clocher de l'église de Villemomble ", L'Architecture, 1927, vol. XL, no 8, p. 233-240.

Albert Louvet, "L'église du Saint-Esprit, à Paris ", L'Architecture, 15 octobre 1934, p. 355-372.

Abbé Mancel, Église Sainte-Thérèse de l'Enfant-Jésus d'Élisabethville, 1928, [1930].

Philippe Ollier, Paul Tournon. Architecture religieuse (1881-1964), mémoire de DEA d'Histoire de l'art, Bruno Foucart dir., université de Paris IV-Sorbonne, 2004.

Paul Tournon, architecte, 1881-1964, Institut de France, Académie des Beaux-Arts, 1969, catalogue d'exposition, Musée de Caen.

Giorgio Pigafetta, Antonella Mastrorilli, Paul Tournon architecte (1881-1964). Le "Moderniste sage ", Mardaga, Sprimont, 2004.

Henri Pingusson, "Paul Tournon ", Cahier du Syndicat des architectes de la Seine, no 46, 1965.

Jeanne de la Ruwière, "L'architecture religieuse moderne", Clarté, $5^{\mathrm{e}}$ année, $\mathrm{n}^{\mathrm{o}}$ 9, septembre 1932, p. 1-6.

Carlo Sarrabezolles, "La sculpture sans maquette par taille directe du béton en prise ", Bulletin de la Société d'encouragement de l'industrie nationale, octobre 1933, p. 529-534.

[Miriam Simon], Église du Saint-Esprit, http://equipement.paris.fr/Église_du_Saint-Esprit

Simon Texier, "L'Église du Saint-Esprit ", dans Gilles-Antoine Langlois (dir.), Le XII ${ }^{e}$ arrondissement, Paris, association artistique de la Ville de Paris, 1996, p. 192-199.

Simon Texier (dir.), Églises parisiennes du XX $X^{e}$ siècle, Association Artistique de la Ville de Paris, Paris, 1996

Micheline Tissot, Byzance à Paris, Lys Éditions Amatteis, Le Mée-sur-Seine, 2005.

Marion Tournon-Branly, Paul Tournon architecte, 1881-1964, Éd. Dominique Vincent, 1976.

Thomas Vivier, "Le sacerdoce du béton armé ", Le Moniteur architecture, AMC, n 73 , septembre 1996, p. 48-52. 